\title{
Three-dimensional boundary integral modeling of spontaneous earthquake sequences and aseismic slip
}

\author{
Nadia Lapusta ${ }^{1,2}$ and Yi Liu ${ }^{1}$ \\ Received 17 July 2008; revised 8 January 2009; accepted 21 May 2009; published 16 September 2009.
}

[1] Fault processes involve complex patterns of seismic events and aseismic slip. This work develops a three-dimensional (3-D) methodology for simulating long-term history of spontaneous seismic and aseismic slip on a vertical planar strike-slip fault subjected to slow tectonic loading. Our approach reproduces all stages of earthquake cycles, from accelerating slip before dynamic instability, to rapid dynamic propagation of earthquake rupture, to postseismic slip, and to interseismic creep, including aseismic transients. We use the developed 3-D methodology to study interaction of fault slip with a small patch of higher normal stress over long-term slip history. For uniform initial prestress, dynamic rupture is significantly affected by the stronger patch in the first simulated event but not in subsequent ones. The change in behavior is due to redistribution of shear stress by prior slip, which demonstrates that distributions of fault strength and stress are related and illustrates the importance of simulating long slip histories even in studies of dynamic rupture. Despite no long-term effect on dynamic rupture, the small patch of higher normal stress influences nucleation processes and hence long-term slip patterns in the model. Comparison of the fully dynamic simulations and a widely used quasi-dynamic approach shows that the quasi-dynamic approach modifies long-term slip patterns in addition to resulting in much smaller slip velocities and rupture speeds during dynamic events. We show that the response of quasi-dynamic formulations with reduced radiation damping terms can be scaled to match the results of the standard quasi-dynamic formulation and hence cannot improve the comparison.

Citation: Lapusta, N., and Y. Liu (2009), Three-dimensional boundary integral modeling of spontaneous earthquake sequences and aseismic slip, J. Geophys. Res., 114, B09303, doi:10.1029/2008JB005934.

\section{Introduction}

[2] Fault processes involve both seismic events or earthquakes and complex patterns of quasi-static aseismic slip. Understanding physics and mechanics of this behavior in its entirety is a fascinating scientific problem. However, even for the more pragmatic goal of understanding only the behavior of destructive large dynamic events, it is still important to consider the entire earthquake cycle, since aseismic slip may determine where earthquakes would nucleate as well as modify stress and other initial conditions before dynamic rupture. Modeling long-term slip histories of faults is quite challenging because of the variety of temporal and spatial scales involved. Slow loading requires hundreds to thousands of years in simulated time and fault zone dimensions are in tens to hundreds of kilometers. At the same time, rapid changes in stress and slip rate at the

\footnotetext{
${ }^{1}$ Division of Engineering and Applied Science, California Institute of Technology, Pasadena, California, USA.

${ }^{2}$ Division of Geological and Planetary Sciences, California Institute of Technology, Pasadena, California, USA.

Copyright 2009 by the American Geophysical Union. 0148-0227/09/2008JB005934\$09.00
}

propagating dynamic rupture tip occur over distances of order meters and times of order a small fraction of a second.

[3] Several approaches to modeling long-term histories of fault slip have been proposed [e.g., Shibazaki and Mastsu'ura, 1992; Cochard and Madariaga, 1996; Kato, 2004; Duan and Oglesby, 2005; Liu and Rice, 2005; Hillers et al., 2006; Ziv and Cochard, 2006] but all of them adopted simplified treatments of either slow tectonic loading and hence aseismic slip, or inertial effects during dynamic rupture, or transition between interseismic periods and dynamic rupture. Lapusta et al. [2000], on the basis of prior studies [Tse and Rice, 1986; Rice and Ben-Zion, 1996; Ben-Zion and Rice, 1997], developed a methodology capable of capturing both seismic and aseismic slip and the gradual process of earthquake nucleation. However, the model of Lapusta et al. [2000] is two-dimensional (2-D) and neglects variations in the along-strike fault dimension. Therefore, it cannot be directly compared to observations and cannot be used to study a number of important problems such as interaction of fault slip with compact fault heterogeneities. In 2-D models, the fault is simplified to a line, and any heterogeneity in stress or friction properties blocks the entire fault. In 3-D models, the fault is represented as a surface that can include local heterogeneities and complex patterns of frictional and other properties. 
[4] In this work, we develop a 3-D methodology for simulating long-term history of spontaneous seismic and aseismic slip on a vertical planar strike-slip fault subjected to laboratory-derived rate and state friction and slow tectonic loading. The algorithm is able to resolve all stages of earthquake cycle in detail, including gradual nucleation processes, dynamic rupture propagation, postseismic slip, and aseismic processes throughout the loading period. Our approach builds on the model of Lapusta et al. [2000], with a number of modifications required in three dimensions such as a different truncation procedure, and uses a boundary integral method. The dynamic part of our 3-D methodology has been validated through the Southern California Earthquake Center (SCEC) code comparison exercise and additional studies [Day et al., 2005; Harris et al., 2008].

[5] Our methodology is described in section 2. Section 3 illustrates its potential by presenting simulations of longterm slip on a fault segment with relatively simple distributions of fault properties. The slip response of the fault model combines a range of seismic and aseismic phenomena. That example is used to explore the effect of several physical and numerical parameters (sections 3 and 4). We then consider two more application examples that demonstrate (1) the importance of conducting long-term simulations even if the main emphasis is on the behavior of dynamic rupture (section 5) and (2) the necessity of including inertial effects in long-term simulations of slip (section 6).

[6] In section 5, we consider how fault slip interacts with compact heterogeneity in the form of a patch of higher normal stress over many earthquake cycles. This kind of problem cannot be considered in a 2-D fault model. Such patches can result on natural faults from slight local nonplanarity of the fault surface. Three-dimensional simulations of single dynamic events suggest that such fault heterogeneities can strongly influence the development of dynamic ruptures, e.g., inducing supershear rupture speeds [e.g., Dunham et al., 2003; Liu and Lapusta, 2008]. However, in simulations of single dynamic events, specified initial conditions, such as initial shear stress, have a determining effect on the resulting dynamic rupture. Our methodology allows us to simulate the interaction of slip with heterogeneity under conditions that naturally develop in the model due to prior seismic and aseismic slip, and to compare that evolved behavior with the one due to arbitrarily chosen initial conditions. We do find significant differences between dynamic rupture behavior in the first and subsequent events, demonstrating the importance of simulating long-term slip histories.

[7] Section 6 compares the fully dynamic formulation developed in this work with quasi-dynamic approaches [e.g., Rice, 1993], which have been widely used in earthquake studies [e.g., Rice, 1993; Ben-Zion and Rice, 1995; Rice and Ben-Zion, 1996; Hori et al., 2004; Kato, 2004; Hillers et al., 2006; Ziv and Cochard, 2006]. Quasi-dynamic approaches significantly simplify the treatment of inertial effects during simulated earthquakes by ignoring wavemediated stress transfers. Results of our comparison underscore the importance of including full inertial effects. We also explore the possibility of improving the standard quasi- dynamic formulation by decreasing the radiation damping term, as suggested by Lapusta et al. [2000].

[8] Our findings are summarized in section 7.

\section{Methodology}

\subsection{Spectral Representation of Elastodynamic Relations}

[9] We extend the spectral boundary integral method [Perrin et al., 1995; Geubelle and Rice, 1995; Ben-Zion and Rice, 1997; Lapusta et al., 2000; Day et al., 2005] to 3-D simulations of long deformation history of a planar strike-slip fault. Consider two identical elastic half-spaces separated by a planar fault interface. The fault coincides with the $y=0$ plane of a Cartesian coordinate system $x y z$. Let us define displacement discontinuities $\delta_{i}(x, z ; t), i=x, y$, $z$ on the fault $y=0$ as $\delta_{i}(x, z ; t)=u_{i}\left(x, y=0^{+}, z ; t\right)-u_{i}(x, y=$ $\left.0^{-}, z ; t\right)$, where $u_{i}(x, y, z ; t)$ are displacement components along the $i$ direction. In the following discussion, we refer to tangential displacement discontinuities $\delta_{x}$ and $\delta_{z}$ as components of slip and to the time derivative of tangential displacement discontinuities $V_{x}$ and $V_{z}, V_{i}(x, z ; t)=\partial \delta_{i}(x$, $z ; t) / \partial t, i=x, z$, as components of slip velocity. In this study, we consider problems that do not involve opening $\left(\delta_{y}(x, z ; t)=0\right)$ and contain only slips $\delta_{x}$ and $\delta_{z}$.

[10] The tractions $\tau_{y i}(x, z ; t) \equiv \tau_{i}(x, z ; t), i=x, y, z$ on the interface $y=0$ can be expressed as

$$
\tau_{i}(x, z ; t)=\tau_{i}^{o}(x, z ; t)+f_{i}(x, z ; t)-\eta_{i} V_{i}(x, z ; t) .
$$

In the elastodynamic relation (1), $\eta_{x}=\eta_{z}=\mu /\left(2 c_{\mathrm{s}}\right)$ and $\eta_{y}=$ $\mu c_{\mathrm{p}} /\left(2 c_{\mathrm{s}}^{2}\right)$, where $\mu$ is the shear modulus and $c_{\mathrm{p}}$ and $c_{\mathrm{s}}$ are the pressure and shear wave speeds, respectively; $\tau_{i}^{o}(x, z ; t)$ are loading tractions, i.e., tractions that would be induced on the fault due to external loading if the fault were restricted against any displacement discontinuities; and $f_{i}(x, z ; t)$ account for wave-mediated stress transfers. The terms $-\eta_{i} V_{i}(x, z ; t)$ represent radiation damping [Rice, 1993; Cochard and Madariaga, 1994]. If there is no opening of the interface, or $\delta_{y}(x, z ; t)=0$ for all $x, z$, then $f_{y}(x, z ; t)$ vanishes and $\tau_{y}(x, z ; t)=\tau_{y}^{o}(x, z ; t)$ according to equations (1). This means that slips $\delta_{x}$ and $\delta_{z}$ on a planar fault separating identical elastic solids do not alter normal traction on the fault and, without opening, the normal traction is only determined by the external loading.

[11] The linear functionals $f_{i}(x, z ; t), i=x, z$ incorporate much of the elastodynamic response and, in the space-time formulation, involve convolution integrals of $\delta_{i}(x, z ; t), i=x$, $x, z$ over space variables $x, z$ and time $t$ [e.g., Cochard and Madariaga, 1994; Perrin et al., 1995]. In the spectral representation, $f_{i}(x, z ; t)$ and $\delta_{i}(x, z ; t)$ are expressed as Fourier series in space, truncated for the purpose of numerical computation [Geubelle and Rice, 1995]:

$$
\begin{aligned}
& \delta_{i}(x, z ; t)=\sum_{k=-N_{x} / 2}^{N_{x} / 2} \sum_{m=-N_{z} / 2}^{N_{z} / 2} D_{i}(k, m ; t) \exp \left(2 \pi i\left(\frac{k x}{\lambda_{x}}+\frac{m z}{\lambda_{z}}\right)\right), \\
& f_{i}(x, z ; t)=\sum_{k=-N_{x} / 2}^{N_{x} / 2} \sum_{m=-N_{z} / 2}^{N_{z} / 2} F_{i}(k, m ; t) \exp \left(2 \pi i\left(\frac{k x}{\lambda_{x}}+\frac{m z}{\lambda_{z}}\right)\right)
\end{aligned}
$$


In equations (2), $\lambda_{x}$ and $\lambda_{z}$ are the sizes of the fault domain under consideration, which is periodically repeated along both $x$ and $z$ directions. The fault domain is discretized into $N_{x}$ by $N_{z}$ square cells with the side $\Delta x$ :

$$
\Delta x=\lambda_{x} / N_{x}=\lambda_{z} / N_{z}
$$

Let us define the wave vector $\hat{\mathbf{q}}=(\hat{k}, \hat{m})$ as

$$
\hat{k}=2 \pi k / \lambda_{x}, \hat{m}=2 \pi m / \lambda_{z}, \hat{q}=|\hat{\mathbf{q}}|=\sqrt{\hat{k}^{2}+\hat{m}^{2}} .
$$

Then the Fourier coefficients $F_{i}(k, m ; t)$ and $D_{i}(k, m ; t)$, $i=x, z$, are related by the following expressions:

$$
\begin{aligned}
\left\{\begin{array}{l}
F_{x}(k, m ; t) \\
F_{z}(k, m ; t)
\end{array}\right\}= & -\frac{\mu}{2 \hat{q}}\left[\begin{array}{cc}
\hat{k}^{2} & \hat{m} \hat{k} \\
\hat{m} \hat{k} & \hat{m}^{2}
\end{array}\right]\left\{2\left(1-\frac{c_{\mathrm{s}}^{2}}{c_{\mathrm{p}}^{2}}\right)\left(\begin{array}{c}
D_{x}(k, m ; t) \\
D_{z}(k, m ; t)
\end{array}\right)\right. \\
& \left.-\int_{0}^{t} K_{\mathrm{II}}\left(\hat{q} c_{\mathrm{s}}\left(t-t^{\prime}\right)\right)\left(\begin{array}{c}
\dot{D}_{x}\left(k, m ; t^{\prime}\right) \\
\dot{D}_{z}\left(k, m ; t^{\prime}\right)
\end{array}\right) d t^{\prime}\right\} \\
& -\frac{\mu}{2 \hat{q}}\left[\begin{array}{cc}
\hat{m}^{2} & -\hat{m} \hat{k} \\
-\hat{m} \hat{k} & \hat{k}^{2}
\end{array}\right]\left\{\left(\begin{array}{c}
D_{x}(k, m ; t) \\
D_{z}(k, m ; t)
\end{array}\right)\right. \\
& \left.-\int_{0}^{t} K_{\mathrm{III}}\left(\hat{q} c_{\mathrm{s}}\left(t-t^{\prime}\right)\right)\left(\begin{array}{l}
\dot{D}_{x}\left(k, m ; t^{\prime}\right) \\
\dot{D}_{z}\left(k, m ; t^{\prime}\right)
\end{array}\right) d t^{\prime}\right\},
\end{aligned}
$$

where $K_{\mathrm{II}}(\rho)$ and $K_{\mathrm{III}}(\rho)$ are integral kernels for mode II and III problems, respectively. These kernels have analytical expressions given in Appendix A. The relations (5) reflect the well-known fact that the general 3-D shear problem involves a combination of mode II and mode III slip.

\subsection{Truncation of Elastodynamic Response}

[12] Elastodynamic response expressed by the convolution integrals in (5) can be truncated for problems that involve long deformation histories with short periods of fast slip. During slow, interseismic periods, the deformation process is quasi-static and there is no need to keep track of inertial effects. Truncation procedures for antiplane (mode III) problems were given by Ben-Zion and Rice [1997] and Lapusta et al. [2000]. Here we develop the appropriate modifications for our 3-D model.

[13] To implement the truncation, we compute the convolutions in (5) from $t-T_{w}$ to $t$ only, where $T_{w}$ is the truncation time window. Convolution kernels $K_{\mathrm{II}}(\rho)$ and $K_{\text {III }}(\rho)$ oscillate with decaying amplitude for larger $\rho$ (Appendix A). Slip velocities, and hence their Fourier coefficients $\dot{D}_{x}$ and $\dot{D}_{z}$, are near zero for most of the fault history, except during simulated earthquakes, which have durations of the order of the time for the shear wave to propagate through the simulated domain. Hence, following Lapusta et al. [2000], $T_{w}$ is expressed in the form

$$
T_{w}=\alpha \lambda / c_{\mathrm{s}}
$$

where $\lambda$ is the largest extent of the seismogenic zone and $\alpha$ is a truncation parameter of order 1 . We call this truncation scheme "frequency-independent," as the truncation window does not depend on the frequency of Fourier modes. Larger truncation windows make the problem closer to the one without truncation but they also increase the computational expense. In our simulations, we find that the choice $\alpha=1$ gives results that do not change for larger truncation windows (section 4.2).

[14] The truncation procedure can be made more efficient by making the truncation window $T_{w}$ dependent on Fourier modes. Fourier coefficients for higher frequencies are generally smaller. Even more importantly, the kernel argument $\rho=\hat{q} c_{\mathrm{s}}\left(t-t^{\prime}\right)$ scales with the mode frequency $\hat{q}$ and, for larger $\hat{q}$, the same time window corresponds to longer integration intervals in terms of the kernel argument $\rho$. Since the kernels oscillate with decaying amplitude for larger $\rho$, one can limit the length of kernel windows, making the corresponding time windows shorter.

[15] The truncation procedure and parameters used by Lapusta et al. [2000] resulted in approximately $T_{w}(\hat{q}) \propto$ $\hat{q}^{-1}$. That scheme was efficient and accurate for 2-D antiplane (mode III) problems considered by Lapusta et al. [2000], but in 3-D problems, we find that it produces much lower slip velocities and rupture speeds than frequencyindependent truncation. This is because both mode III and mode II kernels are involved in the 3-D formulation and the mode II kernel is more oscillatory and decays slower than the mode III kernel. From Appendix A, for $\rho \gg 1, K_{\text {II }} \sim$ $O\left(\rho^{-1 / 2}\right)$, while $K_{\text {III }} \sim O\left(\rho^{-3 / 2}\right)$.

[16] We have developed a new scheme that truncates less at low frequencies than the scheme and parameter choices of Lapusta et al. [2000]. For low-frequency modes, the time windows are constant, $T_{w}(\hat{q})=\alpha \lambda / c_{\mathrm{s}}$, as in the frequencyindependent truncation, with the kernel windows increasing for those modes. However, the kernel windows cannot be larger than a given value $\rho_{c}$, which becomes a truncation parameter. Let us denote by $\hat{q}_{c}$ the frequency at which the kernel windows reach the length $\rho_{c}$. Then the frequencydependent truncation windows expressed in terms of time are given by

$$
T_{w}(\hat{q})= \begin{cases}\alpha \lambda / c_{\mathrm{s}}, & \hat{q} \leq \hat{q}_{c} \\ \alpha \lambda / c_{\mathrm{s}} \hat{q}_{c} / \hat{q}, & \hat{q} \geq \hat{q}_{c} .\end{cases}
$$

Hence, $T_{w}(\hat{q}) \sim \hat{q}^{-1}$ for higher frequencies. Note that the lowest-frequency mode $\hat{\mathbf{q}}=(0,0)$ with $\hat{q}=0$ corresponds to the uniform slip over the fault interface and has no contribution to stress transfers, so we set $T_{w}(0)=0$. In equation (7), $\hat{q}$ varies between the lowest nonzero frequency $\hat{q}_{\text {min }}=2 \pi / \lambda_{x}$ (assuming, without loss of generality, that $\lambda_{x} \geq \lambda_{z}$ ) and the highest-frequency $\hat{q}_{\max }=\sqrt{2} \pi / \Delta x$. In terms of the kernel argument $\rho=\hat{q} c_{\mathrm{s}}\left(t-t^{\prime}\right)$, the time windows (7) correspond to the following kernel windows $\rho_{w}(\hat{q})=\hat{q} c_{\mathrm{s}} T_{w}(\hat{q}):$

$$
\rho_{w}(\hat{q})= \begin{cases}\alpha \lambda \hat{q}, & \hat{q} \leq \hat{q}_{c} \\ \rho_{c}=\alpha \lambda \hat{q}_{c}, & \hat{q} \geq \hat{q}_{c} .\end{cases}
$$

In our simulations, $\rho_{c} \geq 100$ result in the same slip response as that with frequency-independent truncation (section 4.3). Such values of the kernel argument correspond to the kernel amplitudes smaller than 0.0008 for mode III and 0.08 for mode II.

[17] Let us estimate how much more efficient the frequency-dependent truncation is by comparing the memory 
requirements for the two truncation procedures. For simplicity, let us assume that $\lambda=\lambda_{x}=\lambda_{z}$ and $N_{x}=N_{z}=N=\lambda / \Delta x$. $\Delta x$. For convolution computations, each time window $T_{w}(\hat{q})$ is discretized with the time interval $\Delta t_{\min }=\gamma \Delta x / c_{\mathrm{s}}$, where $\gamma$ is a constant parameter discussed in Appendix B, resulting in $N_{T}(\hat{q})$ intervals. For the frequency-independent truncation, this number is given by

$$
N_{T}(\hat{q})=\frac{T_{w}(\hat{q})}{\Delta t_{\min }}=\frac{\alpha N}{\gamma} .
$$

For each frequency mode, we need four arrays of the size $N_{T}(\hat{q})$ to store kernels and Fourier coefficients of slip velocities. The total size of the arrays are

$$
N_{\text {tot }}=\sum_{k=-N / 2}^{N / 2} \sum_{m=-N / 2}^{N / 2} 4 N_{T}(\hat{q})=\frac{4 \alpha N}{\gamma} N^{2} \sim O\left(N^{3}\right)
$$

For the frequency-dependent truncation, the total size of the arrays storing convolution values changes to

$$
\begin{aligned}
N_{\mathrm{tot}}^{\mathrm{mod}}= & \sum_{k=-N / 2}^{N / 2} \sum_{m=-N / 2}^{N / 2} 4 N_{T}(\hat{q}) \approx \int_{-N / 2}^{N / 2} \int_{-N / 2}^{N / 2} 4 N_{T}(\hat{q}) d k d m \\
& <\left(2 \sqrt{2} \rho_{c}-\frac{\rho_{c}^{2}}{\pi \alpha N}\right) \frac{N^{2}}{\gamma} \sim \rho_{c} O\left(N^{2}\right) .
\end{aligned}
$$

For small values of $\rho_{c} \ll \alpha \lambda \hat{q}_{\max }$, the required memory is significantly smaller for the frequency-dependent truncation. To compute the estimate in equation (11), the summation over discrete $k$ and $m$ is replaced by the continuous integration over $d k$ and $d m$; for $N \gg 1$, the error induced by this approximation is negligible. Then, to obtain an upper bound of the resulting integral, the square domain of the integration $[-N / 2, N / 2] \times[-N / 2, N / 2]$ is replaced by a larger circular domain, with the integration variables and their limits being $0 \leq \hat{q} \leq \hat{q}_{\max }$ and $0 \leq \theta<2 \pi$.

\subsection{Fault Constitutive Response: Rate and State Friction Laws}

[18] The fault resistance to sliding is described by laboratory-derived rate and state friction laws which have been widely used to model earthquake phenomena [Dieterich, 1979; Ruina, 1983; Dieterich, 2007, and references therein]. A general form of rate and state friction laws is

$$
\left\{\begin{array}{l}
\tau=\phi(V, \theta, \sigma) \\
\dot{\theta}=\varphi(V, \theta, \sigma)
\end{array}\right.
$$

where $\tau=\sqrt{\tau_{x}^{2}+\tau_{z}^{2}}$ is the magnitude of shear traction vector $\tau=\left(\tau_{x}, \tau_{z}\right), V=\sqrt{V_{x}^{2}+V_{z}^{2}}$ is the magnitude of the slip velocity vector $\boldsymbol{V}=\left(V_{x}, V_{z}\right), \sigma=-\tau_{y}$ is the normal traction (positive in compression), and $\theta$ is the state variable. It has been experimentally established that shear traction instantaneously increases (decreases) in response to a sudden increase (decrease) of slip velocity [e.g., Dieterich, 1979; Ruina, 1983; Marone, 1998], which implies $\partial \phi(V, \theta, \sigma) /$ $\partial V>0$, the feature commonly referred to as the positive direct effect. As discussed by Lapusta et al. [2000], the presence of this instantaneous positive response is essential for the numerical procedure to be able to adopt large time steps during quasi-static deformation processes while yielding stable numerical results.

[19] Several specific forms of rate and state friction laws have been proposed. Here we adopt the aging law [Dieterich, 1979, 1981; Ruina, 1983] in the form appropriate for constant normal stress $\sigma$ :

$$
\begin{gathered}
\tau=\sigma\left(f_{o}+a \ln \frac{V}{V_{o}}+b \ln \frac{V_{o} \theta}{L}\right), \\
\dot{\theta}=1-\frac{V \theta}{L}
\end{gathered}
$$

where $f_{o}$ and $V_{o}$ are the reference friction coefficient and slip velocity, $a>0$ and $b>0$ are rate and state parameters of order 0.01 , and $L$ is the characteristic slip distance. At constant slip velocity $V$, the state variable $\theta$, and hence the shear traction $\tau$, evolve toward steady state values $\theta_{s s}(V)$ and $\tau_{s s}(V)$, respectively, with $\theta_{s s}(V)=L / V$ and $\tau_{s s}(V)=\sigma\left[f_{o}+\right.$ $\left.(a-b) \ln \left(V / V_{o}\right)\right]$. The friction law is said to exhibit steady state velocity strengthening if $a-b>0$, and steady state velocity weakening if $a-b<0$. In equation (13a), $\tau$ is not defined for $V=0$. To remedy that, we use a regularized version of the law [Rice and Ben-Zion, 1996; Ben-Zion and Rice, 1997; Lapusta et al., 2000] described in Appendix B.

[20] Dynamic instability (i.e., an earthquake) is able to develop only if the steady state velocity-weakening region of the fault exceeds the nucleation size $h^{*}$ [e.g., Rice and Ruina, 1983; Rice, 1993; Rubin and Ampuero, 2005]. Two theoretical estimates of the earthquake nucleation size for 2-D problems are given by

$$
h_{\mathrm{RR}}^{*}=\frac{\pi}{4} \frac{\mu^{*} L}{(b-a) \sigma},
$$

$$
h_{\mathrm{RA}}^{*}=\frac{2}{\pi} \frac{\mu^{*} b L}{(b-a)^{2} \sigma},
$$

where $\mu^{*}=\mu$ for mode III and $\mu^{*}=\mu /(1-\nu)$ for mode II. The estimate $h^{*}{ }_{\mathrm{RR}}$ was derived from the linear stability analysis of steady sliding by Rice and Ruina [1983], while $h^{*}{ }_{\text {RA }}$ was obtained for the parameter regime $a / b>0.5$ by Rubin and Ampuero [2005] using the energy balance for a quasi-statically extending crack. Three-dimensional estimates would be larger by a factor of two to three; $h^{*}{ }_{\mathrm{RA}}$ needs to be increased by a factor of $\pi^{2} / 4$ (A. Rubin, private communication, 2008). Chen and Lapusta [2009] have found that the resulting estimate,

$$
h^{*}=\left(\pi^{2} / 4\right) h_{\mathrm{RA}}^{*}
$$

matches nucleation sizes in their 3-D simulations quite well. Hence, we adopt that estimate in our study.

[21] The rate and state friction laws (13) behave similarly to standard linear slip-weakening laws during dynamic 
rupture propagation processes [e.g., Cocco and Bizzarri, 2002]. As dynamic rupture arrives at a point along the fault, slip velocity rapidly increases with negligible slip, leading to $V \theta / L \gg 1$. With that condition, equation (13b) can be approximately rewritten as $d \theta / d t=-V \theta / L$ and integrated to express the state variable in terms of slip. From equation (13a), one then gets

$$
\frac{d \tau}{d t}=\sigma a \frac{d(\ln V)}{d t}-\frac{\sigma b}{L} \frac{d \delta}{d t}
$$

In (17), $V$ is already in the seismic range and $\ln V$ does not change much, making the term $\sigma a d(\ln V) / d t$ much smaller than $(\sigma b / L)(d \delta / d t)$. Hence, equation (17) describes linear decrease of shear stress with slip, with the slip-weakening rate $W$ :

$$
W=-\frac{d \tau}{d \delta} \approx \frac{\sigma b}{L}
$$

During this process, the state variable evolves with slip and the steady state is eventually reached, with shear resistance that has logarithmic dependence on slip velocity and hence does not vary much as long as slip velocity remains in the seismic range. That corresponds to the constant dynamic resistance of linear slip-weakening formulations.

[22] Note that alternative rate and state formulations have been proposed, with different equations for the evolution of the state variable, such as the slip law [Dieterich, 1979, 1981; Ruina, 1983], the combined law of Kato and Tullis [2003], and the law of Perrin et al. [1995]. Recent experiments by Bayart et al. [2006] suggest that the slip law is a better description of the friction response in velocity jump experiments. The methodology developed in this work can be easily adopted to the alternative rate and state formulations, as well as to laws with variable normal stress [Dieterich, 2007] and modified formulations that include more significant weakening at seismic slip rates [Rice, 2006].

\subsection{Criteria for Spatial Discretization}

[23] In numerical simulations, the spatial cell size $\Delta x$ needs to be small enough to capture the model response. A number of studies [Rice, 1993; Ben-Zion and Rice, 1997; Lapusta et al., 2000] proposed that $h^{*}{ }_{\mathrm{RR}}$ is the crucial length scale to resolve in 2-D antiplane earthquake cycle modeling, with $h^{*}{ }_{\mathrm{RR}} / \Delta x$ being an important parameter. The length scale $h^{*}{ }_{\text {RR }}$ gives the critical size of a cell that cannot become unstable on its own on a quasi-statically sliding interface [Rice and Ruina, 1983; Lapusta et al., 2000], and hence, it is an important length scale governing interseismic processes and, in particular, earthquake nucleation.

[24] However, earthquake cycle simulations also involve dynamic rupture, and that introduces another resolution criterion, $\Lambda / \Delta x$, where $\Lambda$ is the cohesive zone size [Palmer and Rice, 1973; Day et al., 2005, and references therein]. The cohesive zone size gives the spatial length scale over which the shear stress drops from its peak to residual values at the propagating rupture front. This length scale controls the numerical resolution during dynamic rupture. Let us denote by $\Lambda_{0}$ the size of $\Lambda$ at the rupture speed $c=0^{+}$ ( $\Lambda$ decreases for larger rupture speeds). For the fault inter- face governed by linear slip-weakening law, $\Lambda_{0}$ can be expressed as [Palmer and Rice, 1973; Day et al., 2005]

$$
\Lambda_{0}=C_{1} \frac{\mu^{*}}{W}
$$

where $W$ is the slip-weakening rate and $C_{1}$ is a constant which is equal to $9 \pi / 32$ if the stress traction distribution within the cohesive zone is linear in space. For the rate-andstate friction laws we use, $W=b \sigma / L$ (sections 2.3 and 3), and hence, $\Lambda_{0}$ is given by

$$
\Lambda_{0}=C_{1} \frac{\mu^{*} L}{b \sigma} .
$$

Through 3-D dynamic rupture simulations, Day et al. [2005] established that $\Lambda_{0} / \Delta x$ of 3 to 5 are required to resolve dynamic rupture.

[25] Hence, the cell size $\Delta x$ has to be small enough to resolve both $\Lambda_{0}$ and $h^{*}{ }_{\mathrm{RR}}$. How are the two resolution criteria related? Ignoring the constants of order 1 , the ratio of the two length scales for the constitute law used in this work is

$$
\Lambda_{0} / h_{\mathrm{RR}}^{*}=(b-a) / b
$$

The typical values are 0.015 to 0.02 for $b$ and 0.002 to 0.004 for $(b-a)$, making the ratio $\Lambda_{0} / h^{*}{ }_{\mathrm{RR}}$ vary from about 0.1 to 0.25 . Hence, the resolution criterion based on $\Lambda_{0}$ dominates for these typical parameters. In this work and in the work by Lapusta et al. [2000], $b=0.019$ and $b-a=0.004$ are used, giving $\Lambda_{0} / h^{*}{ }_{\mathrm{RR}} \approx 0.2$. Hence, resolving $\Lambda_{0}$ with 3 to 5 spatial cells as suggested by Day et al. [2005] corresponds to resolving $h^{*}{ }_{\mathrm{RR}}$ with 15 to 25 cells. This explains why the fully dynamic simulations of earthquake sequences by Lapusta et al. [2000] showed that $h^{*}{ }_{\mathrm{RR}}$ needs to be discretized with about 20 cells in order to obtain resolutionindependent results; that discretization was dictated by the size of the cohesive zone.

[26] Hence, resolving the cohesive zone size $\Lambda_{0}$ is the more stringent requirement for the aging formulation of rate and state friction and typical rate and state parameters. This is true even for quasi-dynamic simulations (section 6), in which $\Lambda_{0}$ needs to be resolved with at least one cell size. For other fault constitutive relations, similar consideration should apply, in that the cell size $\Delta x$ should be small enough to resolve all the relevant length scales in the problem, including the length scale governing the evolution of quasi-static deformation and nucleation of instability as well as the length scale governing the evolution of the rupture front. For different laws, different length scales would dominate the resolution requirements. For example, in a law that combines rate and state friction with a dynamic weakening mechanism, such as pore pressure evolution, either the nucleation length scale or the rupture front length scale may be the smallest one, depending on the values of parameters chosen for the two mechanisms.

\subsection{Computational Procedure}

[27] The response of faults to tectonic loading is characterized by long periods of quasi-static deformation com- 

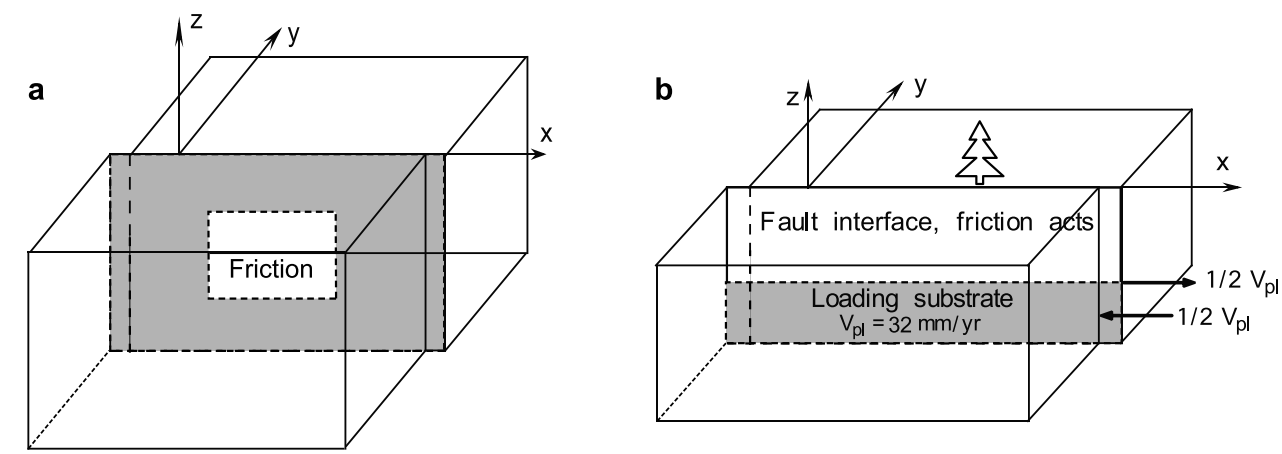

Figure 1. (a) A model of a planar interface embedded in an infinite and homogeneous elastic medium. (b) A vertical strike-slip fault in an elastic half-space. The top part of the fault is governed by rate and state friction, and the bottom part is steadily moving due to tectonic loading.

bined with short bursts of fast slip. To simulate such response, we adopt the variable time stepping of Lapusta et al. [2000], in which the time step is set to be inversely proportional to slip velocity on the fault interface as described in Appendix B. As the result, relatively large time steps, a significant fraction of a year, are used in the interseismic period, while small time steps, a fraction of a second, are used to simulate the evolution of each dynamic rupture. Note that the stability of the stepping procedure relies on the presence of the positive direct effect in the rate and state formulation, the feature that has ample laboratory confirmation.

[28] At each time step, we find updated values of the field variables by equating the elastodynamic tractions on the fault interface represented by equations (1) and the frictional strength of the fault given by equations (13). Appendix B describes the details of the updating procedure. Since 3-D simulations are computationally expensive, parallel coding is an indispensable ingredient in our computations. We use the message passing interface (MPI) techniques to spread the storage of field variables into multiple processors. Calculation of the dynamic response, update of field variables, and fast Fourier transforms (FFTs) are also done in parallel.

\subsection{Model of a Strike-Slip Fault}

[29] The elastodynamic formulation (2)-(5) is valid for a planar interface $y=0$ embedded in an infinite elastic homogeneous medium. Because of the spectral representation, the finite domain of interest is periodically replicated in both $x$ and $z$ directions. Hence, the simulated domain needs to include buffer zones that would prevent dynamic ruptures on each replication from interacting with each other. An example is shown in Figure 1a, where a potentially seismogenic zone (shown in white) is surrounded by the fault region (shown in grey) that can stop dynamic ruptures. Such region can have steady state velocitystrengthening properties and/or prescribed slip velocity (e.g., slip velocity equal to the plate rate or to zero). The methodology developed in this work has been used in such a model to study small repeating earthquakes [Chen and Lapusta, 2009].

[30] Here, we would like to study the behavior of a strikeslip fault embedded in an elastic half-space, with a free surface at $z=0$, as shown in Figure 1b. To fit this model into the formulation (2)-(5), we use the image method [e.g., Lapusta et al., 2000]. The domain that we would like to study on the fault interface is $x \in\left[-\lambda_{x} / 2, \lambda_{x} / 2\right], z \in\left[-\lambda_{z} / 2\right.$, $0]$, where $z=0$ is the free surface. We add a mirror image of that domain by imposing the following conditions in the region $x \in\left[-\lambda_{x} / 2, \lambda_{x} / 2\right], z \in\left[0, \lambda_{z} / 2\right]$ :

$$
\begin{cases}\delta_{x}(x,-z ; t)=\delta_{x}(x, z ; t), & V_{x}(x,-z ; t)=V_{x}(x, z ; t) \\ \delta_{z}(x,-z ; t)=-\delta_{z}(x, z ; t), & V_{z}(x,-z ; t)=-V_{z}(x, z ; t) .\end{cases}
$$

The resulting simulated domain becomes $x \in\left[-\lambda_{x} / 2, \lambda_{x} / z\right]$, $z \in\left[-\lambda_{z} / 2, \lambda_{z} / 2\right]$, and it is that domain that is periodically repeated along both $x$ and $z$ directions to form an infinite interface in a homogeneous elastic space, making the formulation (2)-(5) applicable.

[31] The mirror image method induces the boundary conditions on $z=0$ as $\tau_{z x}=\tau_{z y}=0$ and $u_{z}=0$, which are not exactly the traction free boundary conditions $\tau_{z x}=$ $\tau_{z y}=\tau_{z z}=0$. However, this approximation works quite well for strike-slip faults, which slip mostly in the along-strike direction $x$. In the code comparison exercise organized by the Southern California Earthquake Center (SCEC), simulations of dynamic rupture on a slip-weakening strike-slip fault in an elastic half-space have been compared for different numerical methods [Harris et al., 2004, 2008]. The comparison of our approach with other methods that can represent the true traction-free surface showed that this approximation captures most effects of the free surface and that the errors induced are relatively small and restricted to the region right next to the free surface. Recently, Zhang and Chen [2006a, 2006b] derived a boundary integral formulation with analytical kernels for a planar fault of an arbitrary dipping orientation embedded in an elastic halfspace. These kernels have more complex representations and using them in the context of long-term calculations is a goal for future work.

[32] To incorporate tectonic loading, we assume that the far-field plate motion results in the deeper extension of the fault moving with constant slip rate equal to the plate rate $V_{\mathrm{pl}}$ [Tse and Rice, 1986], as illustrated in Figure 1b. Hence, we assign a constant slip rate $V_{\mathrm{pl}}$ in the corresponding part 

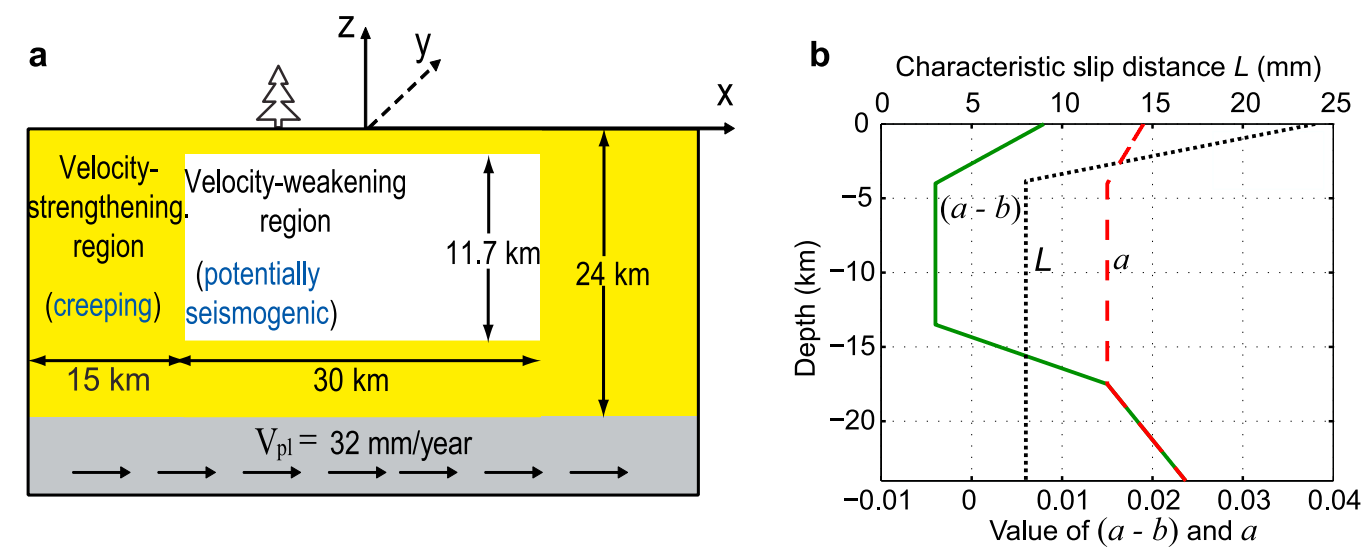

Figure 2. Properties of the simulated fault segment. (a) Rate and state friction acts on the top $24 \mathrm{~km}$ of the fault. A potentially seismogenic region of velocity-weakening properties (shown in white) is surrounded by velocity-strengthening regions (shown in yellow). Below $z=-24 \mathrm{~km}$, steady motion of $32 \mathrm{~mm} / \mathrm{a}$ is imposed. (b) Depth dependence of friction parameters $(a-b), a$, and $L$ in the seismogenic region. The distributions are piecewise linear between the following points: $(a-b)_{|z|=0}=0.008,(a-b)_{|z|=4}=-0.004$, $(a-b)_{|z|=13.5}=-0.004,(a-b)_{|z|=17.5}=0.015,(a-b)_{|z|=24}=0.024, a_{|z|=0}=0.019, a_{|z|=4}=0.015, a_{|z|=17.5}=$ $0.015, a_{|z|=24}=0.024, L_{|z|=0}=24 \mathrm{~mm}, L_{|z|=4}=8 \mathrm{~mm}, L_{|z|=24}=8 \mathrm{~mm}$.

of our domain, and solve for slip rate everywhere else on the fault.

\section{Simulation Example: Fault With a Homogeneous Seismogenic Region}

\subsection{Parameters of the Fault Model}

[33] Let us use the developed methodology to simulate long-term slip history of a strike-slip fault segment which contains a potentially seismogenic region with steady state velocity weakening properties surrounded by steady state velocity-strengthening region (Figure $2 \mathrm{a}$ ). The seismogenic region incorporates gradual variations of rate and state parameters at the top and bottom rheological transitions but we call the region homogeneous in comparison to the model of section 5 where the seismogenic region incorporates a compact heterogeneity in the form of higher normal stress. Model parameters are given in Figure 2 and Table 1. The fault segment is $60 \mathrm{~km}$ long and $30 \mathrm{~km}$ deep. With the mirror image, the simulated domain is $\lambda_{x}=\lambda_{z}=60 \mathrm{~km}$. Rate and state friction acts on the top $24 \mathrm{~km}$ of the fault, while the bottom fault segment, $-30<z<-24 \mathrm{~km}$, slips with the plate rate of $V_{\mathrm{pl}}=10^{-9} \mathrm{~m} / \mathrm{s}$ or $32 \mathrm{~mm} / \mathrm{a}$. The potentially seismogenic velocity-weakening region is located at $-15<$ $x<15 \mathrm{~km}$ and $-14.34<z<-2.67 \mathrm{~km}$ and it is $L_{\text {seis }}=30 \mathrm{~km}$ long and $W_{\text {seis }}=11.7 \mathrm{~km}$ wide. Within the seismogenic region, the depth distributions of rate and state parameters $a$, $(a-b)$, and $L$ are given in Figure 2b. $L$ linearly increases at shallow depths, qualitatively modeling the plausible situation of larger frictional energy resisting sliding at shallow depths due to wider gouge layers or multiple slip surfaces. Normal traction on the fault interface is space- and timeindependent, $\sigma(x, z)=-\tau_{y}(x, z ; t)=-\tau_{y}^{o}(x, z ; t)=50 \mathrm{MPa}$. The constant value of $\sigma$ close to the free surface is chosen for numerical tractability, to explore several issues unrelated to the free surface such as interaction of rupture with heterogeneity over several earthquake cycles (section 5) and quasi-dynamic and other simplified formulations (section 6). In section 3.4, we compare fault behavior with other distributions of $L$ and $\sigma$, including smaller values of $\sigma$ at shallower depths and depth-independent $L$.

[34] The characteristic slip $L$ shown in Figure 2 is equal to $8 \mathrm{~mm}$ over most of the fault; we also use the distribution with twice smaller values of $L$ for comparison, resulting in $L=4 \mathrm{~mm}$ over most of the fault. We consider two values of $L$ to illustrate changes in the fault behavior due to selection of $L$. The chosen values of $L$ result in much larger 3-D estimates of nucleation sizes, $h^{*}=9 \mathrm{~km}$ for $L=8 \mathrm{~mm}$ and $h^{*}=4.5 \mathrm{~km}$ for $L=4 \mathrm{~mm}$, than what would be obtained on the basis of laboratory values of $L=10-100 \mu \mathrm{m}$. Such large values of $h^{*}$ may be realistic under some conditions, e.g., if effective normal stress $\sigma$ is very low due to fluid overpressure [e.g., Liu and Rice, 2005; Suppe and Yue, 2007] or if steady state velocity-weakening properties are close to velocity neutral. We need such large values of $L$ to be able to resolve the dynamic propagation of seismic events that arise in the model. As discussed in section 2.4, $h^{*}$ should be smaller than the size of the velocity-weakening region in order for the model to produce dynamic events.

Table 1. Parameters of Our Simulations

\begin{tabular}{lcc}
\hline \multicolumn{1}{c}{ Parameter } & Symbol & Value \\
\hline Fault length along strike & $\lambda_{x}$ & $60 \mathrm{~km}$ \\
Fault depth & $\lambda_{z} / 2$ & $30 \mathrm{~km}$ \\
Velocity-weakening region, length & $L_{\text {seis }}$ & $30 \mathrm{~km}$ \\
Velocity-weakening region, width & $W_{\text {seis }}$ & $11.7 \mathrm{~km}$ \\
Loading slip rate & $V_{\mathrm{pl}}$ & $32 \mathrm{~mm} / \mathrm{a}$ \\
Shear wave speed & $c_{\mathrm{s}}$ & $3.0 \mathrm{~km} / \mathrm{s}$ \\
Poisson's ratio & $\nu$ & 0.25 \\
Reference slip velocity & $V_{o}$ & $10^{-6} \mathrm{~m} / \mathrm{s}$ \\
$\quad$ and friction coefficient $_{\text {Rate-and-state parameters }}{ }^{\mathrm{a}}$ & $f_{o}$ & 0.6 \\
$\quad$ in the velocity-weakening region $_{\text {Effective normal stress }}{ }^{\mathrm{a}}$ & $b$ & 0.015 \\
Characteristic slip $^{\mathrm{a}}$ & $\sigma$ & 0.019 \\
Cell size & $L$ & $50 \mathrm{MPa}$ \\
Minimum time step & $\Delta x$ & $100 \mathrm{~mm} / 4 \mathrm{~mm}$ \\
\hline
\end{tabular}

${ }^{a}$ The typical value over the velocity-weakening (potentially seismogenic) region is specified. 

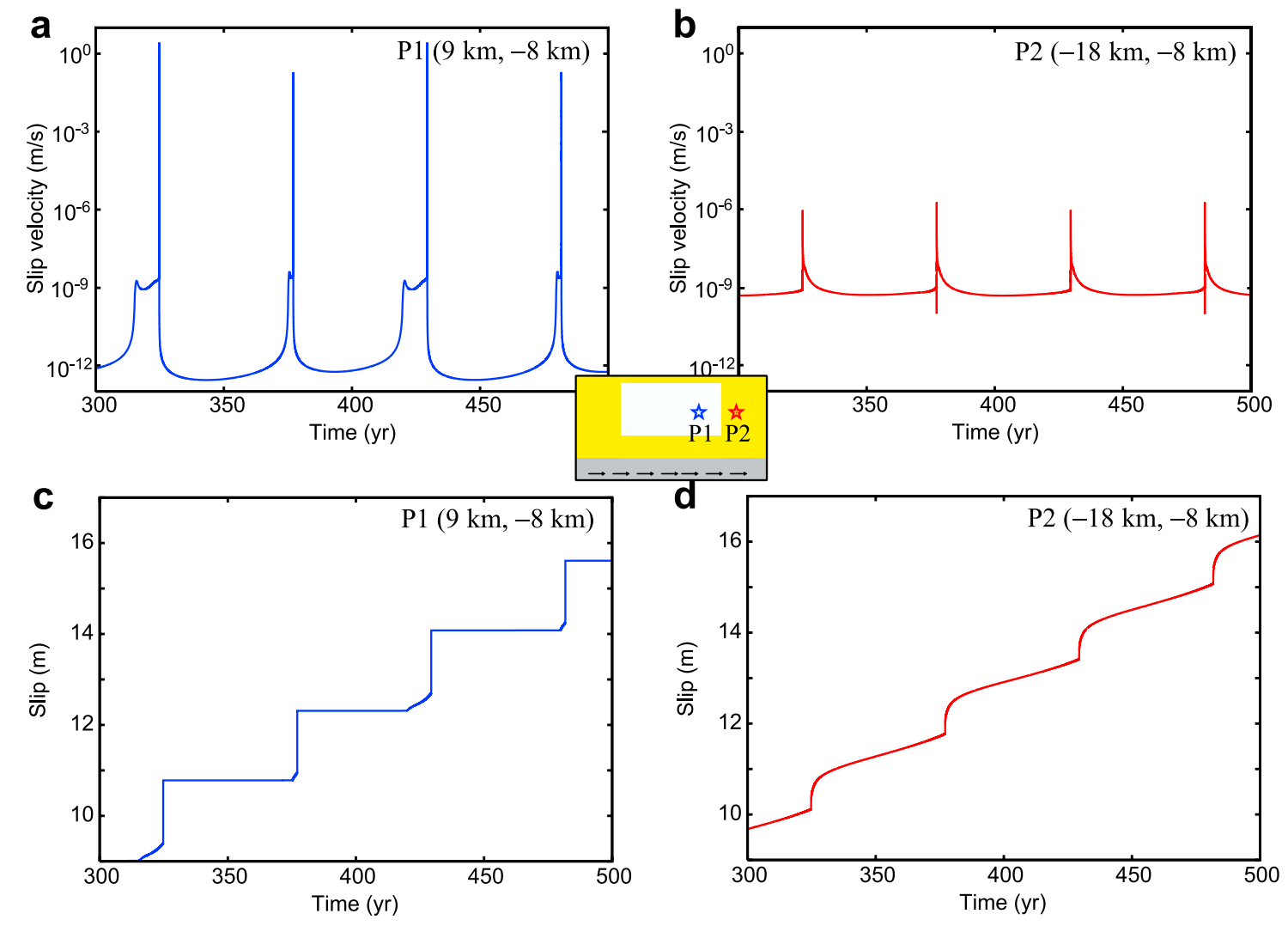

Figure 3. Long-term histories of ( $a$ and b) slip velocity and (c and d) slip at two representative fault locations, P1 from the velocity-weakening region and P2 from the velocity-strengthening region. Slip velocity is plotted on the logarithmic scale. In Figures $3 \mathrm{a}$ and $3 \mathrm{c}$, point P1 $(9 \mathrm{~km},-8 \mathrm{~km})$ exhibits stickslip behavior. It is virtually locked for most of the time (with slip velocity 3 orders of magnitude below the plate rate) but occasionally slips very fast, with maximum slip velocity of the order of $1 \mathrm{~m} / \mathrm{s}$. In Figures $3 \mathrm{~b}$ and d, point P2 $(-18 \mathrm{~km},-8 \mathrm{~km})$ moves throughout the simulated time. After each dynamic event, it has postseismic slip, with maximum slip velocity of the order of $10^{-6} \mathrm{~m} / \mathrm{s}$.

Hence, the parameter $h^{*} / W_{\text {seis }}$ that relates the estimated nucleation size to the width of the velocity-weakening region is an important indicator of how unstable the behavior of the model would be [Liu and Rice, 2005; Rubin, 2008]. $h^{*} / W_{\text {seis }} \geq 1$ predicts quasi-static behavior, while smaller values point to unstable behavior. For $L=8 \mathrm{~mm}, h^{*} /$ $W_{\text {seis }}=0.8$, a value close to 1 . For $L=4 \mathrm{~mm}, h^{*} / W_{\text {seis }}=0.4$, a value appreciably smaller than 1 . Hence, the two cases may exhibit qualitative differences.

[35] The simulation starts with a dynamic event that initiates on the left edge of the fault and propagates through the entire fault. This is achieved by setting higher initial shear stress $\tau_{x}^{o}=1.02 f_{o} \sigma$ for $-15 \mathrm{~km}<x<-10 \mathrm{~km}$ than for the rest of the fault where $\tau_{x}^{o}=1.00 f_{o} \sigma$. The initial values of field quantities affect only the first few events, as the model evolves toward behavior dictated by the model geometry, loading, and friction properties. The fault is discretized into square elements $\Delta x=\Delta z=100 \mathrm{~m}\left(N_{x}=N_{z}=600\right)$ for simulations with $L=8 \mathrm{~mm}$ and $\Delta x=\Delta z=50 \mathrm{~m}\left(N_{x}=N_{z}=\right.$ 1200) for simulations with $L=4 \mathrm{~mm}$. The numerical resolution is discussed in section 4.1. The time step during dynamic events is $\Delta t_{\min }=0.0112 \mathrm{~s}$ for $(L=8 \mathrm{~mm})$ and $\Delta t_{\min }=0.0056 \mathrm{~s}(L=4 \mathrm{~mm})$. Simulations have been done in parallel on 20 processors for $L=8 \mathrm{~mm}$ and 100 processors for $L=4 \mathrm{~mm}$, each with the memory of $2 \mathrm{~GB}$.
About one billion data points are manipulated at each time step for $L=8 \mathrm{~mm}$, and each earthquake cycle requires of the order of 10,000 variable time steps.

\subsection{Fault Response: Dynamic Events and Aseismic Slip, Including Transients}

[36] As expected from stability properties of rate-andstate interfaces, the velocity-strengthening region steadily slips with velocities comparable to the plate rate, while the velocity-weakening region accumulates most of its slip through earthquakes. Histories of slip velocity and slip for two representative points are shown in Figure 3. At point P1 from the velocity-weakening region $(x=9 \mathrm{~km}, z=-8 \mathrm{~km})$, slip velocity is 3 orders of magnitude smaller than the loading plate rate for most of the simulated time, indicating that the fault is typically locked at this location (Figure 3a). Occasionally, slip velocity of P1 goes up to seismic values of the order of $1 \mathrm{~m} / \mathrm{s}$, indicating the occurrence of seismic slip. Slip accumulation of P1 has the corresponding steplike nature (Figure $3 \mathrm{c})$. In contrast, point P2 $(x=18 \mathrm{~km}, z=$ $-8 \mathrm{~km}$ ) from the velocity-strengthening region has slip velocity that never deviates too much from the plate rate of $10^{-9} \mathrm{~m} / \mathrm{s}$ (Figure 3b). Relatively small increases of slip velocity at P2 after each earthquake correspond to post- 

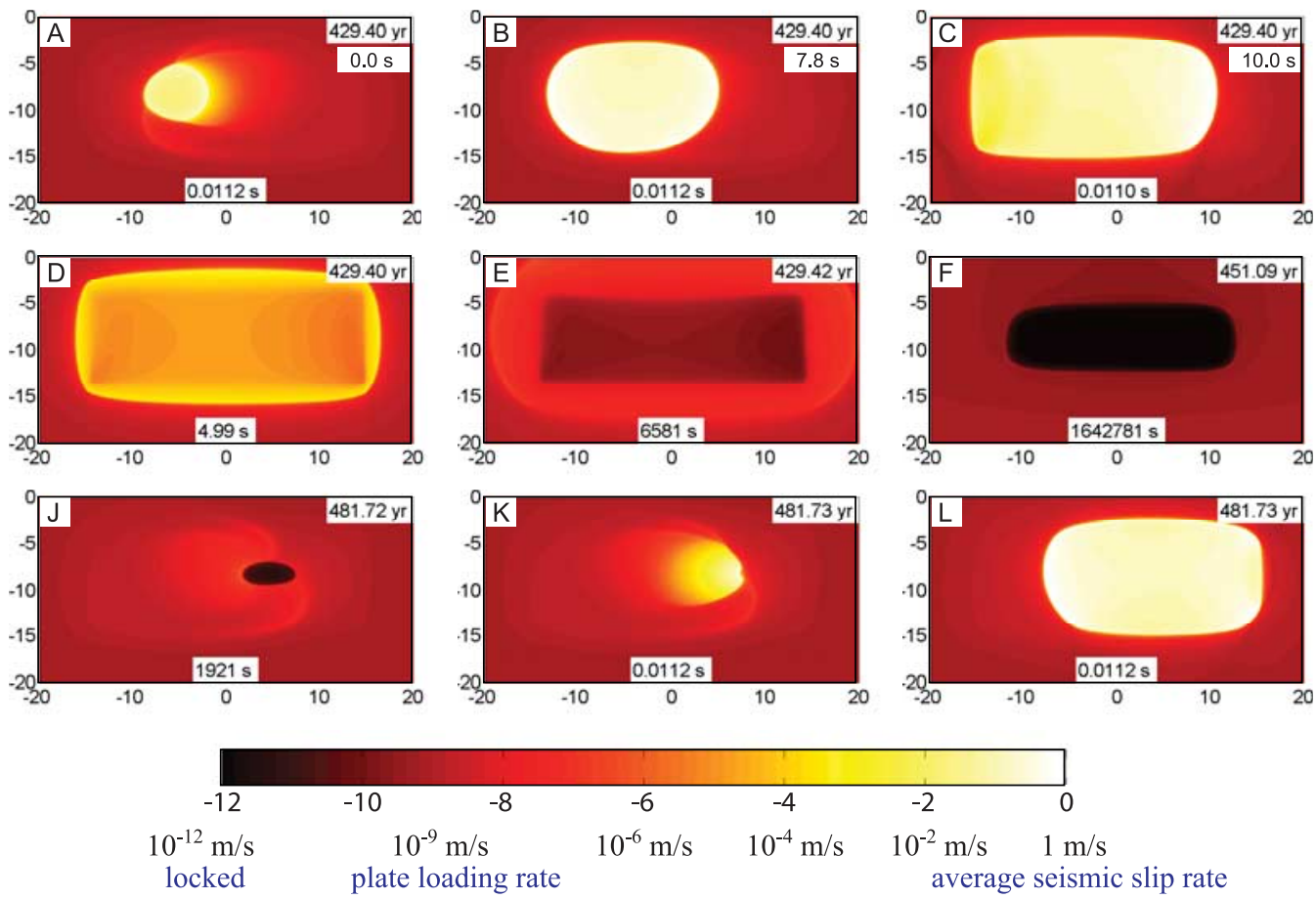

Figure 4. Snapshots of spatial slip velocity distribution during a typical earthquake cycle for $L=8 \mathrm{~mm}$ $\left(h^{*} / W_{\text {seis }}=0.8\right)$. Slip history between the ninth and tenth events is illustrated. Colors represent slip velocity on the logarithmic scale. White and bright yellow correspond to seismic slip rates, orange and red correspond to aseismic slip, and black corresponds to locked portions of the fault. The time $t$ of the snapshot in years (in the top right corner) and the corresponding time step $\Delta t$ in seconds (at the bottom) are shown. Figures $4 a-4 c$ also show the time in seconds elapsed since the time of Figure $4 a$. The simulations reproduce dynamic events (Figures $4 \mathrm{a}-4 \mathrm{c}, 4 \mathrm{k}$ and $4 \mathrm{l}$ ), postseismic slip (Figures $4 \mathrm{~d}$ and $4 \mathrm{e}$ ), and the interseismic period (Figure 4f). Aseismic transient slip occurs between Figures $4 \mathrm{f}$ and $4 \mathrm{j}$, and it is shown in snapshots G8, H8, and I8 of Figure 6 on a different slip velocity scale.

seismic slip. Slip at P2 increases steadily in time, with faster accumulation after each dynamic event (Figure 3d).

[37] Typical earthquake cycles are illustrated in Figures 4 and 5 through snapshots of slip-velocity distribution on the fault at several times between two successive dynamic events. For the case of $L=8 \mathrm{~mm}$ and $h^{*} / W_{\text {seis }}=0.8$ (Figure 4), the ninth earthquake nucleates on the left side of the seismogenic region, and propagates bilaterally first and then mostly to the right (Figures $4 \mathrm{a}-4 \mathrm{c}$ ). The seismic slip causes positive static stress changes in the surrounding velocity-strengthening area, which responds with increased aseismic slip rates that decay over time (Figures $4 \mathrm{~d}$ and $4 \mathrm{e}$ ). This is postseismic slip. During the interseismic period (Figure 4f), the velocity-weakening region is locked, while the surrounding velocity-strengthening region moves with slip velocity of the order of the plate rate. That aseismic slip creates stress concentration at the boundary between the locked and slipping regions, causing slip there and hence continuously moving the boundary into the locked region. For $L=8 \mathrm{~mm}$, the locked region almost disappears (Figure 4j), consistently with the large estimate of the nucleation zone $h^{*}=9 \mathrm{~km}$ which approximates how far slow slip can penetrate into velocity-weakening region without nucleating a dynamic event. That interseismic slip contains a slip episode faster than the plate rate, i.e., an aseismic transient, which is shown in Figure 6 on a different slip velocity scale. The transient develops on the left side of the locked region (snapshot G8) and propagates around the locked region (snapshots G8, H8, and I8), decreasing the locked part in the process. Maximum slip velocity during this aseismic transient is about $10^{-7} \mathrm{~m} / \mathrm{s}$, and its propagation speed is about $8 \mathrm{~km} / \mathrm{a}$. Between the times of 480.43 and 481.52 years, the average slip of the seismogenic region is $0.061 \mathrm{~m}$ and the corresponding moment is $6.4 \times 10^{17} \mathrm{~N} \cdot \mathrm{m}$, an equivalent of a $M_{w}=5.8$ earthquake. These values are consistent, in the order of magnitude sense, with some observations of aseismic transients [e.g., Kawasaki, 2004]. At the end of the transient, the next dynamic event nucleates on the right side of the seismogenic region (Figure $4 \mathrm{k}$ ) and propagates bilaterally first (Figure 4l) and then mainly to the left.

[38] For the smaller value of characteristic slip $L=4 \mathrm{~mm}$ and hence for the smaller $h^{*} / W_{\text {seis }}=0.4$, the seismogenic region is more unstable and experiences less aseismic slip in the interseismic period (Figure 5). Events nucleate closer to the rheological transition (Figure 5a) and propagate more unilaterally (Figures $5 \mathrm{~b}$ and $5 \mathrm{c}$ ). Right after postseismic slip (Figures $5 \mathrm{~d}$ and $5 \mathrm{e}$ ), most of the seismogenic region is locked and the fault behavior for both values of $L$ is quite similar (Figures $4 \mathrm{f}$ and $5 \mathrm{f}$ ). In the interseismic period, aseismic transients still occur for $L=4 \mathrm{~mm}$ (Figure 6, snapshots G4, H4, and I4), but now it is clear that they are mostly confined to the areas of the velocity-weakening 

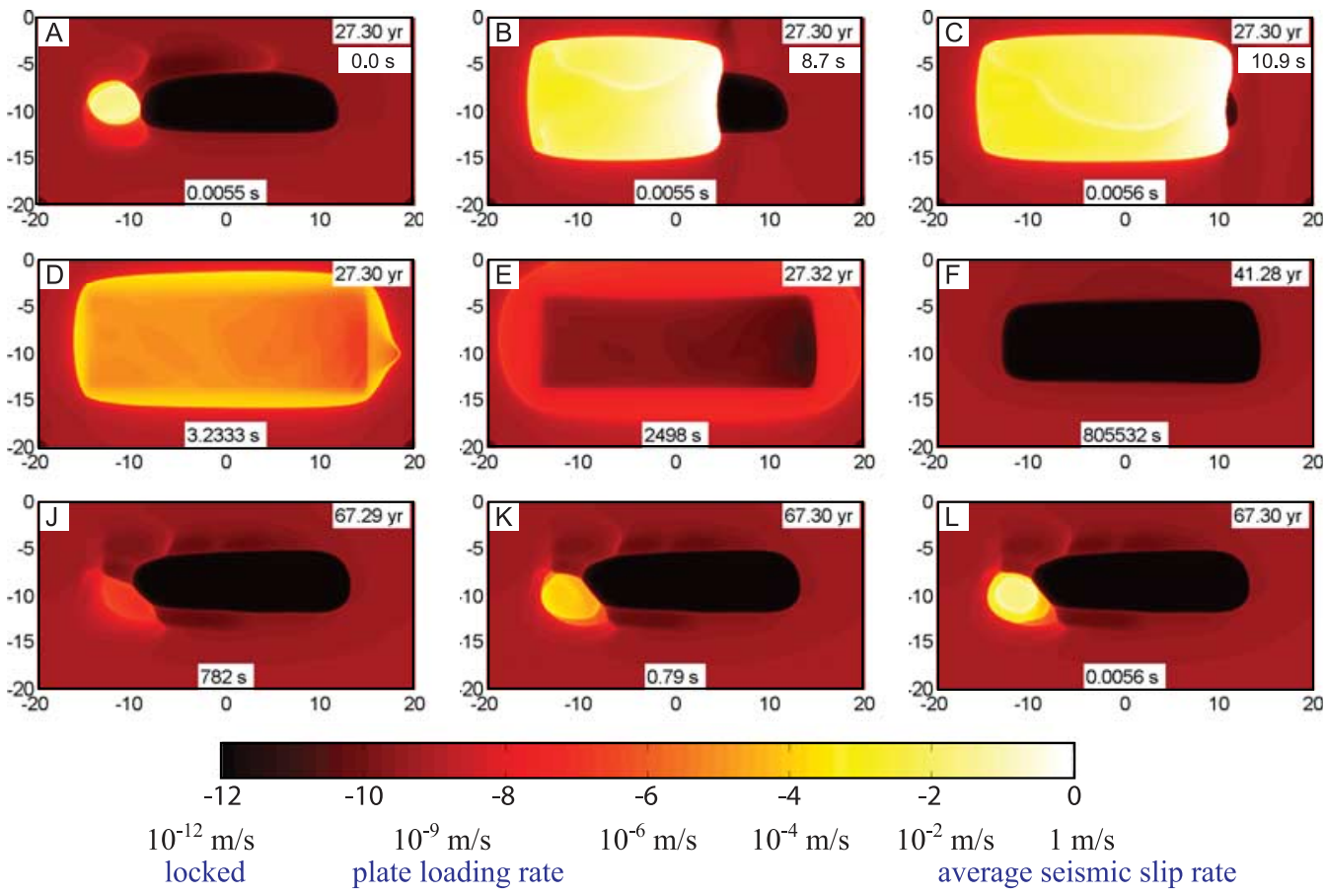

Figure 5. Snapshots of spatial slip velocity distribution during a typical earthquake cycle for $L=4 \mathrm{~mm}$ $\left(h^{*} / W_{\text {seis }}=0.4\right)$. Slip history between the second and third events is illustrated. Colors and time markings have the same meaning as in Figure 4. Compared with the case with $L=8 \mathrm{~mm}$ (Figure 4), dynamic events in the case with $L=4 \mathrm{~mm}$ have smaller nucleation size, nucleate closer to the rheological transition (Figures $5 \mathrm{a}$ and 51), have more unilateral propagation, and develop faster rupture speeds (Figures $5 \mathrm{a}-5 \mathrm{c}$ ). Consistent with the smaller value of $h^{*} / W_{\text {seis }}$, the velocity-weakening region experiences less aseismic slip, with large portion of the region still locked when a seismic event nucleates (Figures $5 \mathrm{a}$ and $5 \mathrm{j}-51$ ). Smaller aseismic transients still occur between Figures $5 \mathrm{f}$ and $5 \mathrm{j}$; they are shown in snapshots $\mathrm{G} 4, \mathrm{H} 4$, and I4 of Figure 6.

region close to rheological transitions that experience slow slip penetration from the nearby velocity-strengthening region. Further discussion of aseismic transients is given in section 7 . When the next dynamic event nucleates (Figures $5 j-51$ ), much of the velocity-weakening region remains locked.

[39] To visualize slip accumulation on the fault through several earthquake cycles, we plot slip along the horizontal line $z=-8 \mathrm{~km}$ (Figure 7). The solid green lines are plotted every 5 years, representing slip accumulations during interseismic periods. The dashed red lines are plotted every 2 seconds when the maximum slip velocity on the fault exceeds $1 \mathrm{~mm} / \mathrm{s}$, illustrating the end of earthquake nucleation and the accumulation of seismic slip. Only a part of the fault, from $x=-20 \mathrm{~km}$ to $x=20 \mathrm{~km}$, is shown. The spacing of the green lines indicates that the fault outside the velocity-weakening region moves steadily for most of the time and experiences faster postseismic motion after dynamic events. Densely spaced red lines correspond to the end of the nucleation phase, while more sparse red lines illustrate dynamic rupture propagation. For $L=8 \mathrm{~mm}$ and $h^{*} / W_{\text {seis }}=0.8$ (Figure 7a), this relatively homogeneous model produces a periodic two-event pattern. For $L=$ $4 \mathrm{~mm}$ and $h^{*} / W_{\text {seis }}=0.4$ (Figure 7b), the model settles into periodic behavior, with all events starting on the left side of the fault. This is because events have larger slip at the other end of the fault and relieve more stress there, resulting in the nucleation of the next event on the same side of the fault.
The behavior is more complex for $h^{*} / W_{\text {seis }}=0.8$ due to aseismic transients. The aseismic transients also always start on the side of the fault that nucleated the previous dynamic event. However, they do not initiate dynamic slip but rather propagate toward the other side of the fault, initiating a dynamic event there.

\subsection{Parameters of Simulated Earthquakes}

[40] The model produces realistic dynamic events, with maximum slip velocity over the fault exceeding $1 \mathrm{~m} / \mathrm{s}$ and rupture speeds reaching $2.5 \mathrm{~km} / \mathrm{s}$. Let us define the seismic moment $M_{0}$ of each event as the moment released on the fault when maximum slip velocity exceeds $0.1 \mathrm{~m} / \mathrm{s}$ :

$$
M_{0}=\int_{t_{\mathrm{ini}}}^{t_{\mathrm{end}}} \int_{\Omega} \mu V\left(x, z ; t^{\prime}\right) d \Omega d t^{\prime},
$$

where $\Omega$ is the seismogenic (velocity-weakening) region with the area $|\Omega|=W_{\text {seis }} L_{\text {seis }}$ and $t_{\text {ini }}$ and $t_{\text {end }}$ are times, for each dynamic event, between which maximum slip velocity on the fault stays larger than $V_{\text {seis }}=0.1 \mathrm{~m} / \mathrm{s}$. Similarly, we compute the static stress drop $\Delta \tau$ as

$$
\begin{aligned}
\Delta \tau & =\bar{\tau}_{x}\left(t_{\text {ini }}\right)-\bar{\tau}_{x}\left(t_{\text {end }}\right) \\
& =\frac{1}{|\Omega|} \int_{\Omega} \tau_{x}\left(x, z ; t_{\text {ini }}\right) d \Omega-\frac{1}{|\Omega|} \int_{\Omega} \tau_{x}\left(x, z ; t_{\text {end }}\right) d \Omega .
\end{aligned}
$$



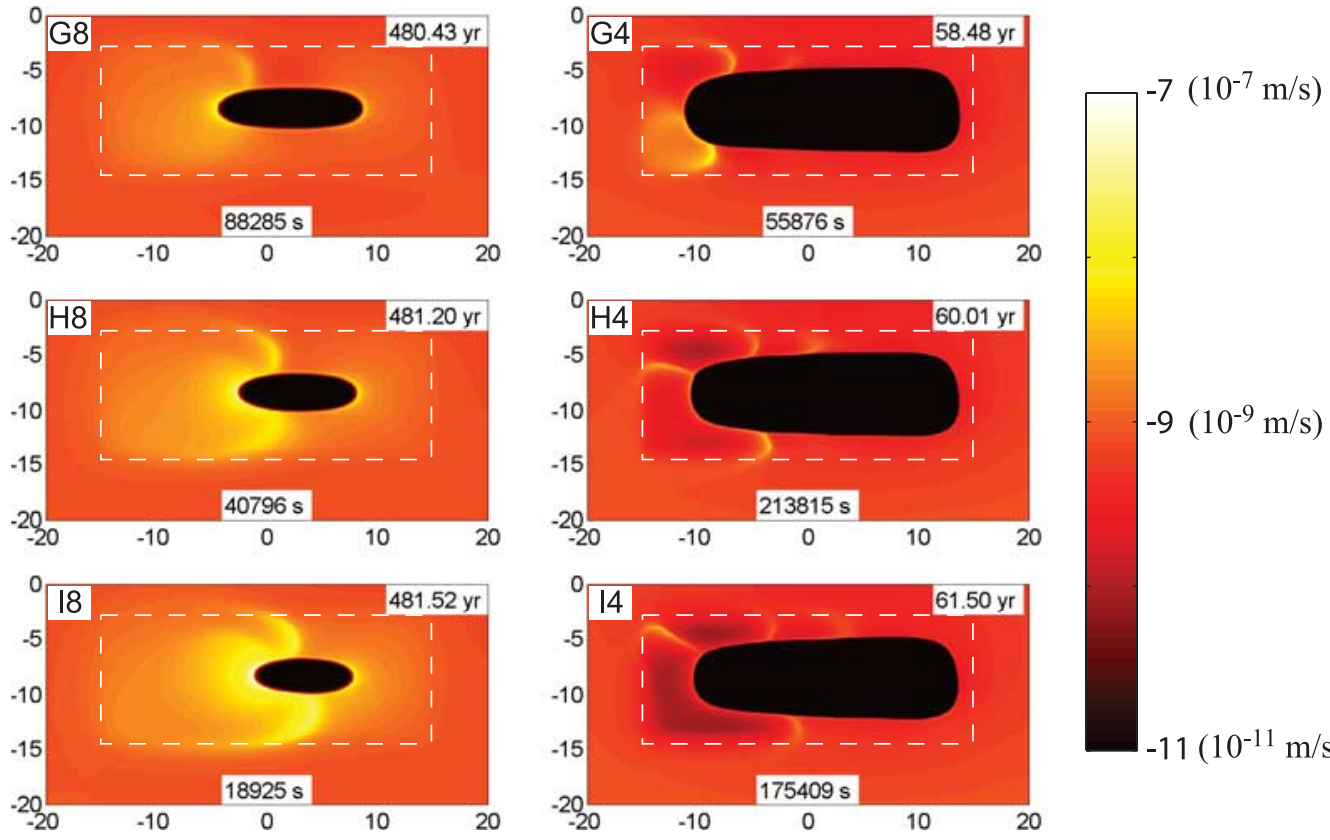

$9\left(10^{-9} \mathrm{~m} / \mathrm{s}\right)$

Figure 6. Snapshots of spatial slip velocity distribution illustrating aseismic transients. White dashed rectangles show the extent of the velocity-weakening region. Snapshots G8, H8, and I8 correspond to $L=$ $8 \mathrm{~mm}$. The aseismic transient travels around the locked portion of the fault. The average rupture speed between snapshots G8 and I8 is of the order of $10 \mathrm{~km} / \mathrm{a}$, and the maximum slip velocity is about $10^{-7} \mathrm{~m} / \mathrm{s}$. The aseismic slip accumulated in the seismogenic region is equivalent to that of a $M_{w}=5.8$ earthquake. Snapshots G4, H4, and I4 correspond to $L=4 \mathrm{~mm}$. The spatial extent of the transients is smaller. Again, the transients travel around the locked portion of the fault. Comparison of the two cases shows that the transients are confined to the band of the velocity-weakening region next to rheological transition which experiences slow slip in the interseismic period. The width of the band scales with the nucleation size and its estimate $h^{*}$ and that is why smaller values of $h^{*} / W_{\text {seis }}$ lead to smaller and more localized aseismic transients.
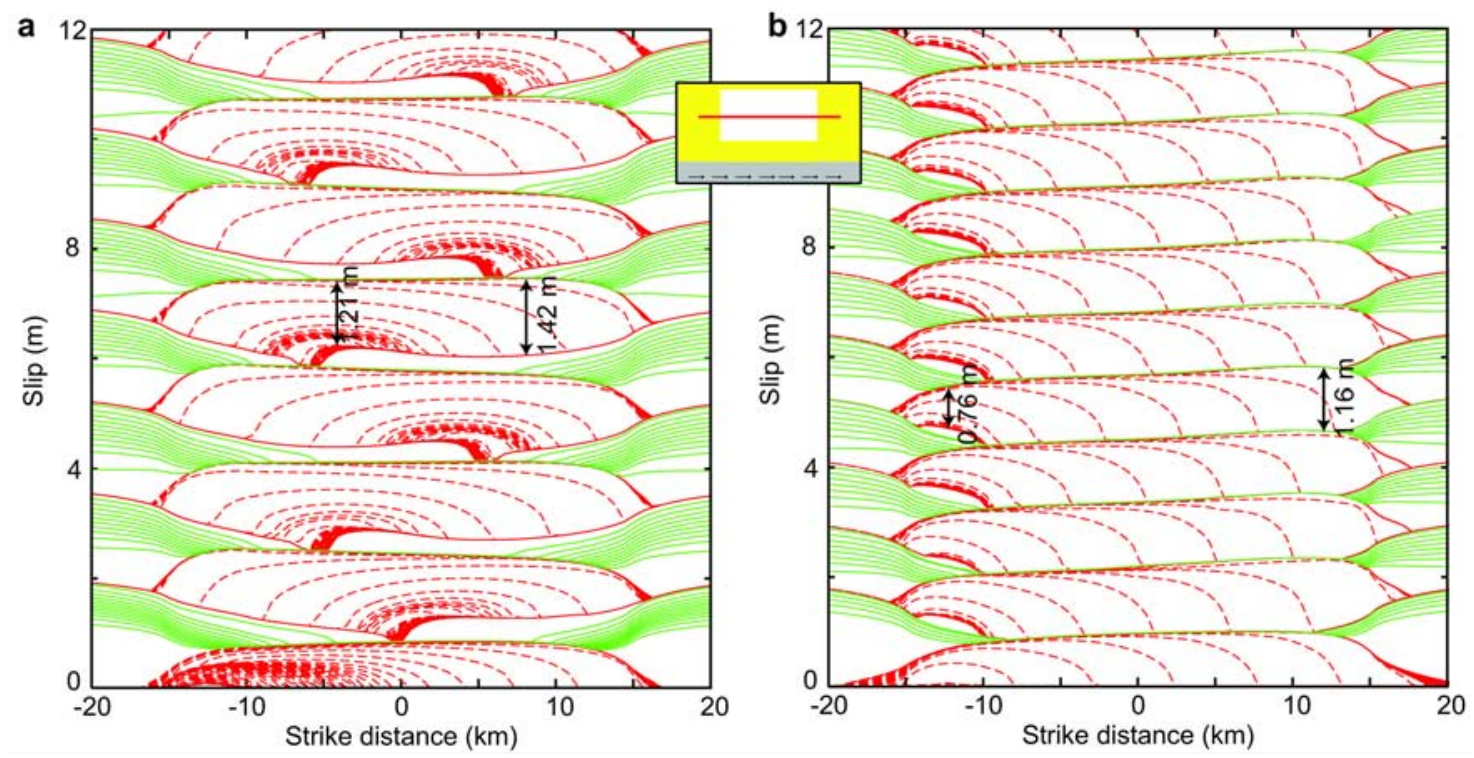

Figure 7. Slip accumulation along the line $z=-8 \mathrm{~km}$ for the case of the homogeneous seismogenic region. Red dashed lines illustrate fast slip; they are plotted every $2 \mathrm{~s}$ when maximum slip velocity over the fault exceeds $1 \mathrm{~mm} / \mathrm{s}$. Green solid lines are plotted every 5 years, representing slip accumulation in interseismic periods. (a) The case with $L=8 \mathrm{~mm}$ settles into periodic two-event pattern. (b) The case with $L=4 \mathrm{~mm}$ results in periodic events. In the latter case, dynamic ruptures propagate faster and are more pulse-like. In both cases, points at the nucleation zones accumulate less slip seismically than points elsewhere on the fault. 

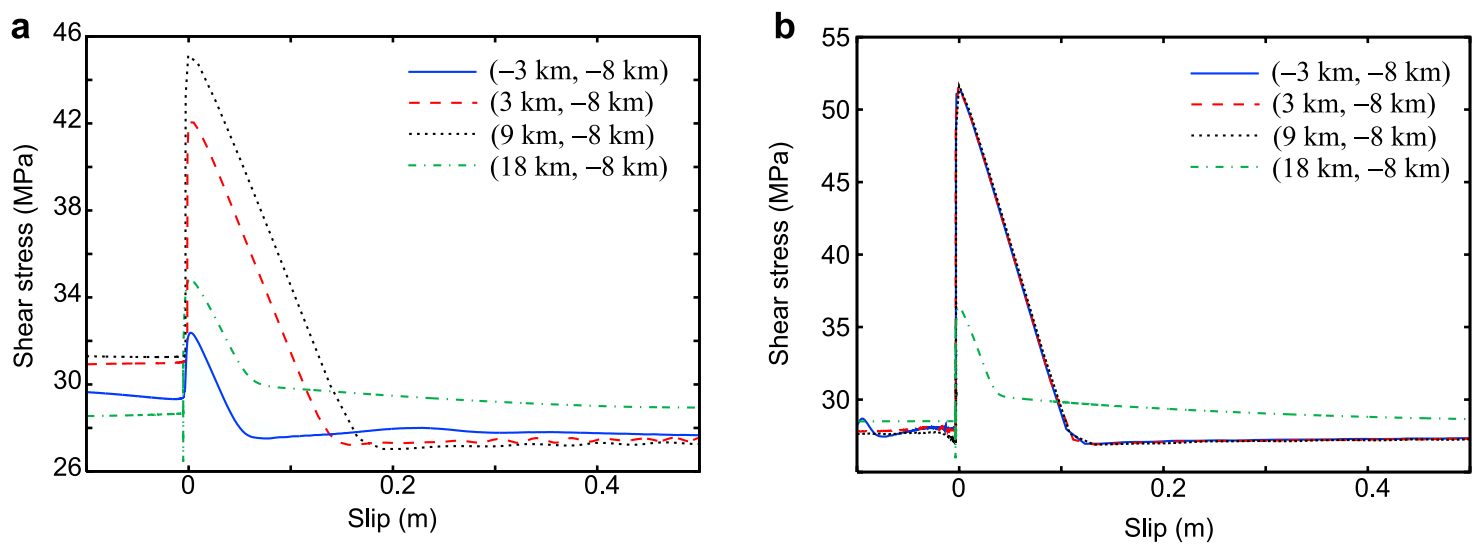

Figure 8. Shear stress as a function of slip during a representative dynamic event (the ninth one in the sequence) for four locations on the fault with (a) $L=8 \mathrm{~mm}$ and (b) $L=4 \mathrm{~mm}$. In both cases, dynamic rupture propagates from the left side of the fault to the right side, passing the velocity-weakening locations $(-3 \mathrm{~km},-8 \mathrm{~km}),(3 \mathrm{~km},-8 \mathrm{~km}),(9 \mathrm{~km},-8 \mathrm{~km})$, and then influencing the velocitystrengthening location $(18 \mathrm{~km},-8 \mathrm{~km})$ as the rupture arrests in the velocity-strengthening region. Zero slip for each point is chosen as the slip when shear stress at the point reaches its peak during the dynamic event. We see that the effective dependence of stress on slip is similar to linear slip-weakening friction, with the slip-weakening rate $W \approx \sigma b / L$. The velocity-strengthening point has a smaller values of $b$ than the other three points and hence a smaller slope. In the case with $L=8 \mathrm{~mm}$, rupture accelerates while propagating through the points shown (Figure 7a), leading to different effective peak strength and slipweakening distances for the three velocity-weakening points. In the case with $L=4 \mathrm{~mm}$, the rupture has nearly reached its limiting speed and it is almost steady (Figure 7b), leading to similar behavior of the velocity-weakening points.

For $L=8 \mathrm{~mm}, M_{0}=1.1 \times 10^{19} \mathrm{~N} \mathrm{~m}$ for each event, corresponding to the moment magnitude $M_{w}=6.6, \bar{\tau}_{x}\left(t_{\text {ini }}\right)=$ 31.2 $\mathrm{MPa}, \bar{\tau}_{x}\left(t_{\text {end }}\right)=27.8 \mathrm{MPa}$, and $\Delta \tau=3.4 \mathrm{MPa}$. For $L=$ $4 \mathrm{~mm}, M_{0}=9.9 \times 10^{18} \mathrm{~N} \mathrm{~m}, M_{w}=6.6, \bar{\tau}_{x}\left(t_{\text {ini }}\right)=31.2 \mathrm{MPa}$, $\bar{\tau}_{x}\left(t_{\text {end }}\right)=28.0 \mathrm{MPa}$, and $\Delta \tau=3.2 \mathrm{MPa}$. The average seismic slip on the seismogenic part of the fault per event is $\bar{\delta}_{\text {seis }}=M_{\text {seis }} / \mu|\Omega|=1.01 \mathrm{~m}$ for $L=8 \mathrm{~mm}$ and $0.94 \mathrm{~m}$ for $L=$ $4 \mathrm{~mm}$. Since the plate loading rate is $V_{\mathrm{pl}}=32 \mathrm{~mm} / \mathrm{a}$ and the earthquake period is $T=52.4$ years for $L=8 \mathrm{~mm}$ and 37.4 years for $L=4 \mathrm{~mm}$, slip accumulation per earthquake cycle is $\bar{\delta}=V_{\mathrm{pl}} T=1.65 \mathrm{~m}$ for $L=8 \mathrm{~mm}$ and $1.18 \mathrm{~m}$ for $L=$ $4 \mathrm{~mm}$. Therefore, $61 \%$ and $80 \%$ of fault slip in the seismogenic region is accumulated seismically for $L=8 \mathrm{~mm}$ and $L=4 \mathrm{~mm}$, respectively.

[41] Each point ruptured dynamically exhibits effective stress slip dependence that closely resembles linear slipweakening laws, as discussed in section 2.3. This is illustrated in Figure 8, which shows the behavior of three velocity-weakening points and one velocity-strengthening point. The velocity-strengthening point is located close to rheological transition. For all curves, the weakening slope is well approximated by $W=-\sigma b / L$. For $L=8 \mathrm{~mm}$, we find that the effective slip-weakening behavior is similar for different points but not identical, with the peak stress and effective slip-weakening distance increasing with the rupture propagation. This is because the rupture accelerates as it propagates along the fault, and the associated increase in peak slip velocity causes increases in the peak stress and effective slip-weakening distance. For $L=4 \mathrm{~mm}$, the dependence of stress on slip is nearly identical for the velocity-weakening points, because the rupture accelerates early in the event and, afterward, the relatively homogeneous fault properties and conditions ensure that the rupture behavior does not change much as the rupture propagates along the fault.

\subsection{Effect of Parameter Distributions Near the Free Surface}

[42] In the presented simulations, effective normal stress $\sigma=50 \mathrm{MPa}$ is constant throughout the fault and the characteristic slip $L$ is depth-dependent near the free surface. Because of the relatively large $\sigma$, and hence large velocitystrengthening effect $\sigma(a-b)$, dynamic rupture arrests shortly upon encountering the shallow velocity-strengthening region and does not reach the free surface (Figure 9a). While constant $\sigma$ at depth can be motivated by fluid overpressure [Rice, 2006], $\sigma$ should decrease to near-zero values at the free surface. To investigate the effect of $\sigma$ and $L$ near the free surface, we consider the case of Lapusta et al. [2000], in which normal stress is depth-dependent near the free surface, $\sigma=\min [2.8+18|z| / \mathrm{km}, 50] \mathrm{MPa}$, and the characteristic slip $L$ is constant and equal to the value at depth. In this case, the rupture propagates all the way to the free surface (Figure 9b), as it did in the 2-D simulations of Lapusta et al. [2000]. However, even with the depthvariable normal stress, we can prevent the rupture from reaching the free surface by modifying rate and state parameters $a$ and $b$ so that $a \sigma$ and $b \sigma$ are the same as in our original example (Figure 9c). For the problems considered in this work, it is not essential whether slip does or does not propagate to the free surface, and we use the 

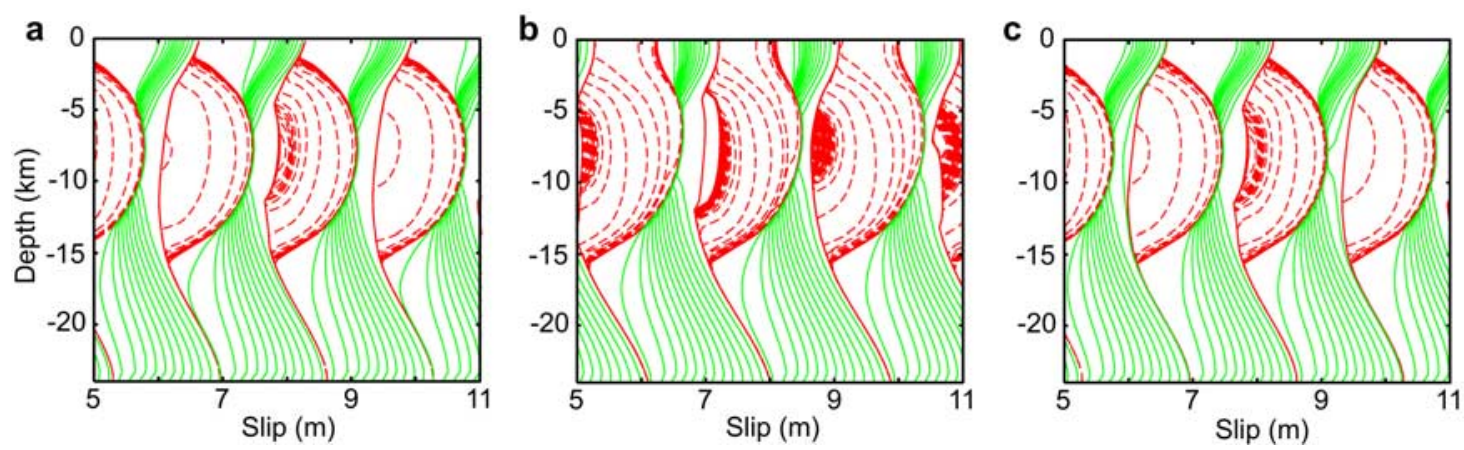

Figure 9. Slip accumulation along the line $x=3 \mathrm{~km}$ for the fault with the homogenous seismogenic region and the case of $L=8 \mathrm{~mm}$. Lines have the same meaning as in Figure 7. Different near-surface parameter distributions are explored. (a) In the case of sections 3.1 and 3.2 and Figures 3-8 with constant normal stress and depth-dependent $L$, dynamic events do not reach the free surface, arresting in the velocity-strengthening region. The free surface accumulates large slip deficit, which is compensated by aseismic slip. (b) For depth-independent $L$ and normal stress decreasing toward the free surface (the same distributions as in the 2-D model of Lapusta et al. [2000]), dynamic ruptures propagate all the way to the free surface, consistently with the results of Lapusta et al. [2000]. (c) When distributions of parameters $a$ and $b$ in the case of Figure $9 \mathrm{~b}$ are modified to match the distributions of $\sigma a$ and $\sigma b$ of the case in Figure 9a, the near-surface behavior becomes very similar to the case of Figure 9a.

parameters of section 3.1 in simulations presented in the following.

\section{Parameter Validation}

\subsection{Spatial Discretization}

[43] For the model with $L=8 \mathrm{~mm}$, we have $h^{*}{ }_{R R}=1.26 \mathrm{~km}$ and $\Lambda_{0}=233 \mathrm{~m}$ for the mode II direction; this theoretical estimate of $\Lambda_{0}$ is close to the value of $300 \mathrm{~m}$ obtained in our simulations. Hence, as discussed in section $2.4, \Lambda_{0}=233 \mathrm{~m}$ is the smaller length scale and the one we should aim to resolve. To make sure our simulations produce resolutionindependent results, we run a series of simulations with cell sizes $\Delta x=50 \mathrm{~m}, 100 \mathrm{~m}, 200 \mathrm{~m}$, and $400 \mathrm{~m}$. Figures $10 \mathrm{a}$ and $10 \mathrm{~b}$ show slip accumulation along $z=-8 \mathrm{~km}$ for $\Delta x=50 \mathrm{~m}$ and $\Delta x=400 \mathrm{~m}$. Comparing with the results for $\Delta x=100 \mathrm{~m}$ in Figure 7 , we find that $\Delta x=50 \mathrm{~m}$ and $\Delta x=100 \mathrm{~m}$ produce virtually indistinguishable slip patterns over earthquake cycles. The value $\Delta x=400 \mathrm{~m}$ produces a completely different slip pattern, indicating
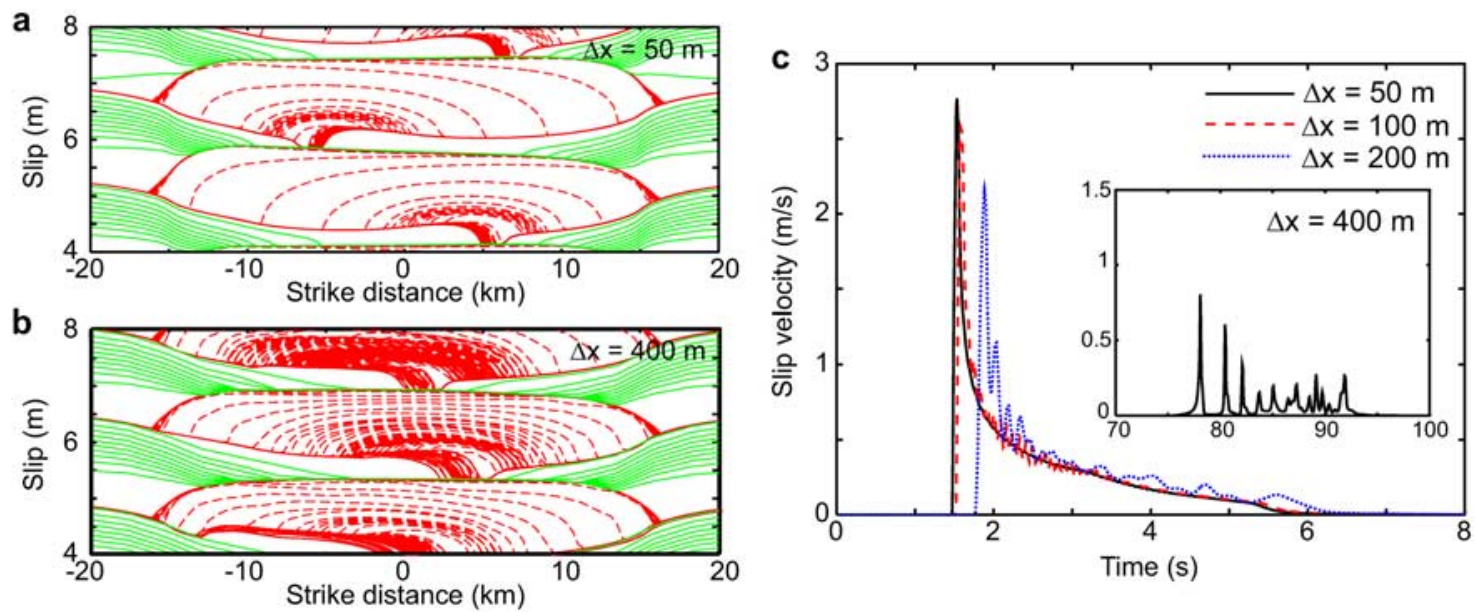

Figure 10. Fully dynamic simulations with different cell sizes $\Delta x$. (a and b) Slip accumulation along the line $z=-8 \mathrm{~km}$ for $\Delta x=50 \mathrm{~m}$ and $400 \mathrm{~m}$, respectively. The results can be compared with Figure $7 \mathrm{a}$ that shows slip accumulation for $\Delta x=100 \mathrm{~m}$. (c) Slip velocity history of the fault location $(9 \mathrm{~km},-8 \mathrm{~km})$ during the fifth event for $\Delta x=50 \mathrm{~m}, 100 \mathrm{~m}, 200 \mathrm{~m}$, and $400 \mathrm{~m}$. Zero in time corresponds to rupture arrival at the location $(6 \mathrm{~km},-8 \mathrm{~km})$. The values $\Delta x=50 \mathrm{~m}$ and $100 \mathrm{~m}$ are both several times smaller than the quasi-static cohesive zone size $\Lambda_{0}=300 \mathrm{~m}$ and produce resolution-independent results. $\Delta x=$ $200 \mathrm{~m}$ provides less adequate resolution, and $\Delta x=400 \mathrm{~m}$ leads to very different results. The numerical resolution in our simulations is dictated by the cohesive zone size, as the nucleation size $h^{*}$, another important length scale, is several times larger. 

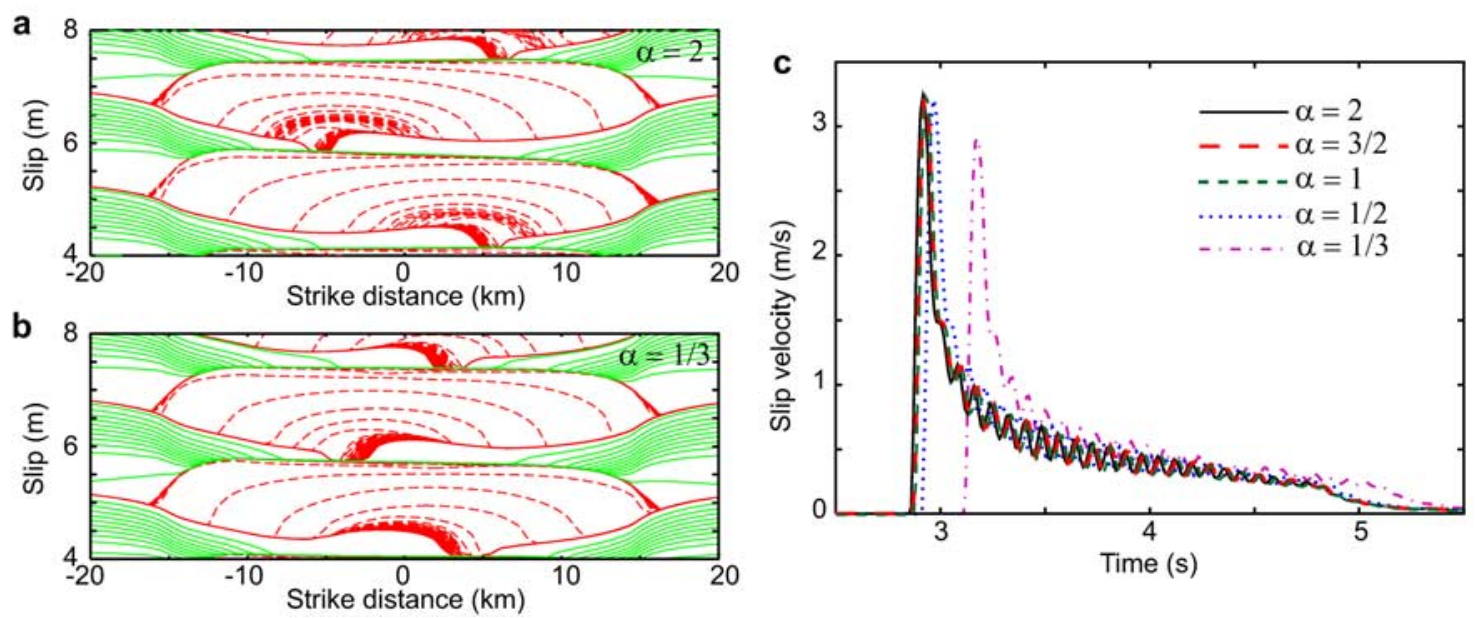

Figure 11. Fully dynamic simulations with different values of the truncation parameter $\alpha$. (a and b) Slip accumulation along the line $z=-8 \mathrm{~km}$ for $\alpha=2$ and $1 / 3$, respectively. The results can be compared with Figure 7a that shows slip accumulation for $\alpha=3 / 2$. (c) Slip velocity history of the fault location (12 km, $-8 \mathrm{~km}$ ) during the fifth event for $\alpha=1 / 3,1 / 2,3 / 2,1$, and 2. Zero in time corresponds to rupture arrival at the location $(6 \mathrm{~km},-8 \mathrm{~km})$. Larger values of $\alpha$ lead to inclusion of longer slip histories in the dynamic response calculation; $\alpha=2,3 / 2$, and 1 produce similar results, while $\alpha=1 / 2$ and $1 / 3$ cause differences as discussed in the text.

poor numerical resolution. The simulation with $\Delta x=200 \mathrm{~m}$ (not shown) produces a slip pattern which is similar to that of $\Delta x=50 \mathrm{~m}$ and $\Delta x=100 \mathrm{~m}$ but has notable differences, such as deeper nucleation regions and $2 \%$ smaller slip per cycle.

[44] Figure 10c shows slip velocity history of the fault location $(9 \mathrm{~km},-8 \mathrm{~km})$ during the fifth event. Zero time is chosen as the arrival time of rupture at the point $(6 \mathrm{~km}$, $-8 \mathrm{~km}$ ). Again, $\Delta x=100 \mathrm{~m}$ and $\Delta x=50 \mathrm{~m}$ produce similar results during dynamic rupture propagation, although slip velocity has some oscillations for $\Delta x=100$. These oscillations are due to the kernel discretization, as discussed in Appendix A. For $\Delta x=200$, rupture time is noticeably larger, slip velocities are smaller, and the profile is more oscillatory. As the rupture propagates, the cohesive zone becomes smaller, making $\Delta x=200 \mathrm{~m}$ inadequate. For $\Delta x=$ $400 \mathrm{~m}$ (insert), the result is completely different, indicating numerical problems. Hence, we confirm the finding of Day et al. [2005] that the quasi-static cohesive zone in 3-D simulations needs to be resolved by about three cells.

\subsection{Frequency-Independent Truncation}

[45] To determine the suitable value of parameter $\alpha$ in the frequency-independent truncation window $T_{w}=\alpha \lambda / c_{\mathrm{s}}$, we do a series of simulations with $\alpha=2,3 / 2,1,1 / 2$, and $1 / 3$. Simulations with $\alpha=2,3 / 2,1$ all produce virtually indistinguishable results in terms of both earthquake patterns and slip velocity histories during individual events (Figures 11 and $7 \mathrm{a}$ ), indicating that either of them can be used in the truncation procedure. We use $\alpha=3 / 2$ in most of our simulations. Simulations with $\alpha=1 / 2$ and $1 / 3$ produce somewhat different behavior. For example, the rupture speed is $2 \%$ smaller for $\alpha=1 / 2$ and $9 \%$ smaller for $\alpha=1 / 3$.

\subsection{Frequency-Dependent Truncation}

[46] As discussed in section 2.2, frequency-dependent truncation can save a lot of computational resources, including memory. It has two parameters, $\alpha$ and $\rho_{c}$. We fix $\alpha=3 / 2$, a value that performs well in the frequencyindependent truncation, and compare results for several values of $\rho_{c}$. For $N_{x}=N_{z}=600$ and $\Delta x=100 \mathrm{~m}$, frequency-independent truncation implies $\rho_{c}=\alpha \lambda \hat{q}_{\max } \approx$ 2000. To activate frequency dependence, we need to choose a smaller value of $\rho_{c}$. We try $\rho_{c}=250,200,150,100,50$, 25 , and $3 \pi / 2$. The last value approximately reproduces the truncation scheme used by Lapusta et al. [2000]. We find that simulations that use frequency-dependent truncation with $\rho_{c} \geq 100$ produce the same results as simulations with frequency-independent truncation. Figure 12 shows slip velocity history of the fault location $(12 \mathrm{~km},-8 \mathrm{~km})$ during the first event for different values of $\rho_{c}$. The simulation with $\rho_{c}=100$ has the same rupture time at this location as the simulation with frequency-independent truncation, and the difference in peak velocity between the two simulations is less than 5\%. Hence, truncation with $\rho_{c}=100$ gives adequate results while using only $9.7 \%$ of the memory required for frequency-independent truncation. The memory savings would be more significant for smaller cell sizes or larger fault dimensions. The simulation with $\rho=\alpha \pi=3 \pi / 2$, which worked well in 2-D antiplane models [Lapusta et al., 2000], produces rather poor results, with much slower rupture speeds and slip velocity. The difference between 2-D antiplane and 3-D problems arises due to properties of mode II kernel as discussed in section 2.2.

\section{Long-Term Interaction of Slip With Compact Heterogeneity}

[47] As an application example, let us investigate longterm behavior of a fault segment in the presence of compact heterogeneity. This study requires the fully dynamic 3-D approach for simulating long-term slip developed in this work, as the existing 2-D dynamic and 3-D quasi-dynamic methodologies may not be able to capture all features of the 


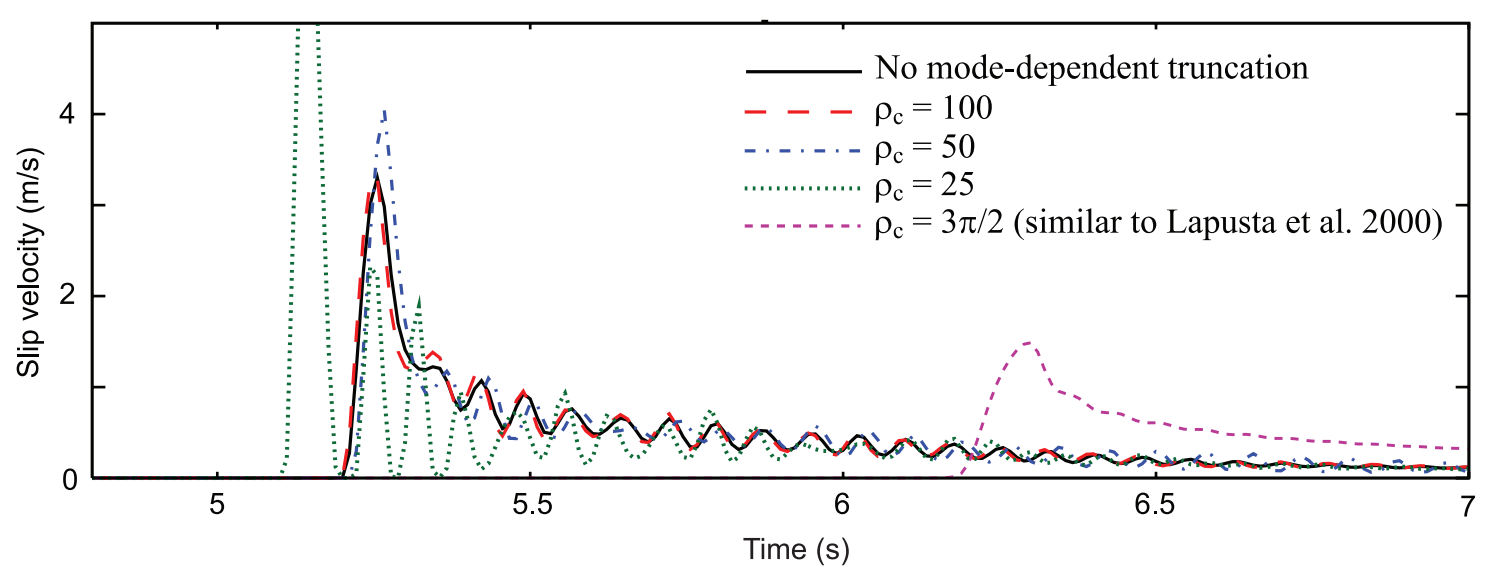

Figure 12. Slip velocity history of the fault location $(12 \mathrm{~km},-8 \mathrm{~km})$ during the first event in fully dynamic simulations with different values of the truncation parameter $\rho_{c}$. Zero in time corresponds to the rupture arrival at the location $(3 \mathrm{~km},-8 \mathrm{~km})$. Larger values of $\rho_{c}$ make the frequency-dependent truncation closer to the frequency-independent one. Our frequency-dependent truncation with $\rho_{c}=3 \pi / 2$ approximately corresponds to the truncation parameters of Lapusta et al. [2000]; $\rho_{c} \geq 100$ produce the same results as the frequency-independent truncation.

response as explained in section 1 . We use the model of section 3.1 with a stronger circular patch of $20 \%$ larger effective normal stress $\sigma$. The patch is centered at the location $(x=3 \mathrm{~km}, z=-8 \mathrm{~km})$ and has the radius of $1 \mathrm{~km}$. The other model parameters are the same as in section 3.1 , including $L=8 \mathrm{~mm}$, with the exception of initial shear stress outside the strip $-15 \mathrm{~km}<x<-10 \mathrm{~km}$. The value $1.00 f_{o} \sigma$ of section 3 results in the first event that is slower than subsequent events (Figure 7a). Since we would like to compare interaction of dynamic rupture with the stronger patch in the first event with the interaction in subsequent events, it is important for the first event to be more dynamic. To achieve that, we use initial shear stress of $1.01 f_{o} \sigma$ outside the strip $-15 \mathrm{~km}<x<-10 \mathrm{~km}$.

\subsection{Supershear Burst in the First Event}

[48] The first event nucleates in the region of higher initial shear stress on the left side of the seismogenic region and propagates toward the location of the patch. Since the patch is stronger than the surrounding fault but the initial shear stress is uniform, the front of dynamic rupture is delayed at the asperity during the first event (Figure 13, top left). Note that the slip velocity scale in Figure 13 is different than in previous figures with slip velocity snapshots; the scale in Figure 13 is chosen to illuminate the rupture front. Slip in the surrounding areas concentrates shear stress at the patch, breaking it and creating a supershear burst over a part of the rupture front (Figure 13, to middle and top right). Along the horizontal line $z=-8 \mathrm{~km}$, the rupture front advances $3.4 \mathrm{~km}$ in $0.84 \mathrm{~s}$, with the average rupture speed of $c=4.0 \mathrm{~km} / \mathrm{s}$, which is larger than the shear wave speed $c_{\mathrm{s}}=3 \mathrm{~km} / \mathrm{s}$. Such supershear bursts were studied by Dunham et al. [2003] in simulations of single earthquakes on faults governed by linear slip-weakening friction. In our simulation, the supershear part of the front transitions back to sub-Rayleigh speed shortly afterward.

\subsection{No Supershear Burst in Subsequent Events}

[49] The supershear burst observed in the first event is due to interaction of dynamic rupture with the normal stress heterogeneity. Will this interaction repeat in subsequent events? The second earthquake also nucleates on the left side of the seismogenic region and propagates toward the stronger patch. Figure 13 (bottom) shows the snapshots of slip velocity distribution during the second event as the rupture front passes through the patch. The snapshots show that there is no interaction of the rupture front with the stronger patch. In Figure 13 (top), corresponding to the first event, the patch location is obvious; in Figure 13 (bottom), corresponding to the second event, we need to mark the patch with a red circle to indicate its location. The rupture front smoothly propagates through the patch with subRayleigh speeds. The average horizontal rupture speed in the time period between the first and the third snapshot is $c=2.0 \mathrm{~km} / \mathrm{s}$ or about 0.7 of the Rayleigh wave speed. Rupture continues to accelerate as it propagates further along the fault interface. Note that the concave rupture front profile in Figure 13 (bottom) is also present in the case without the patch during the second event, and it is not related to the presence of the stronger patch. Rather, it is caused by higher rupture speeds close to the boundary between creeping and locked regions due to shear stress concentration there.

[50] Hence, dynamic rupture does not "notice" the patch during the second event and, in fact, during all subsequent events. This is due to redistribution of shear stress on the fault after the first event. Figure 14 shows two shear stress distributions along the horizontal line $z=-8 \mathrm{~km}$, which passes through the center of the patch, during the first and second events. The distributions correspond to the time when the rupture front that propagates toward the patch is about $2 \mathrm{~km}$ away from the patch. Before rupture propagates through the patch in the first event, shear stress inside the patch is approximately equal to the initial shear stress of 30.3 MPa. The ratio of shear stress and effective normal stress $\tau / \sigma$ is 0.505 inside the patch and 0.606 in the surrounding region. Therefore, the patch delays the rupture and then produces a supershear burst. However, before rupture propagation over the patch in the second event, shear stress inside the patch is higher, about $37.2 \mathrm{MPa}$, and 

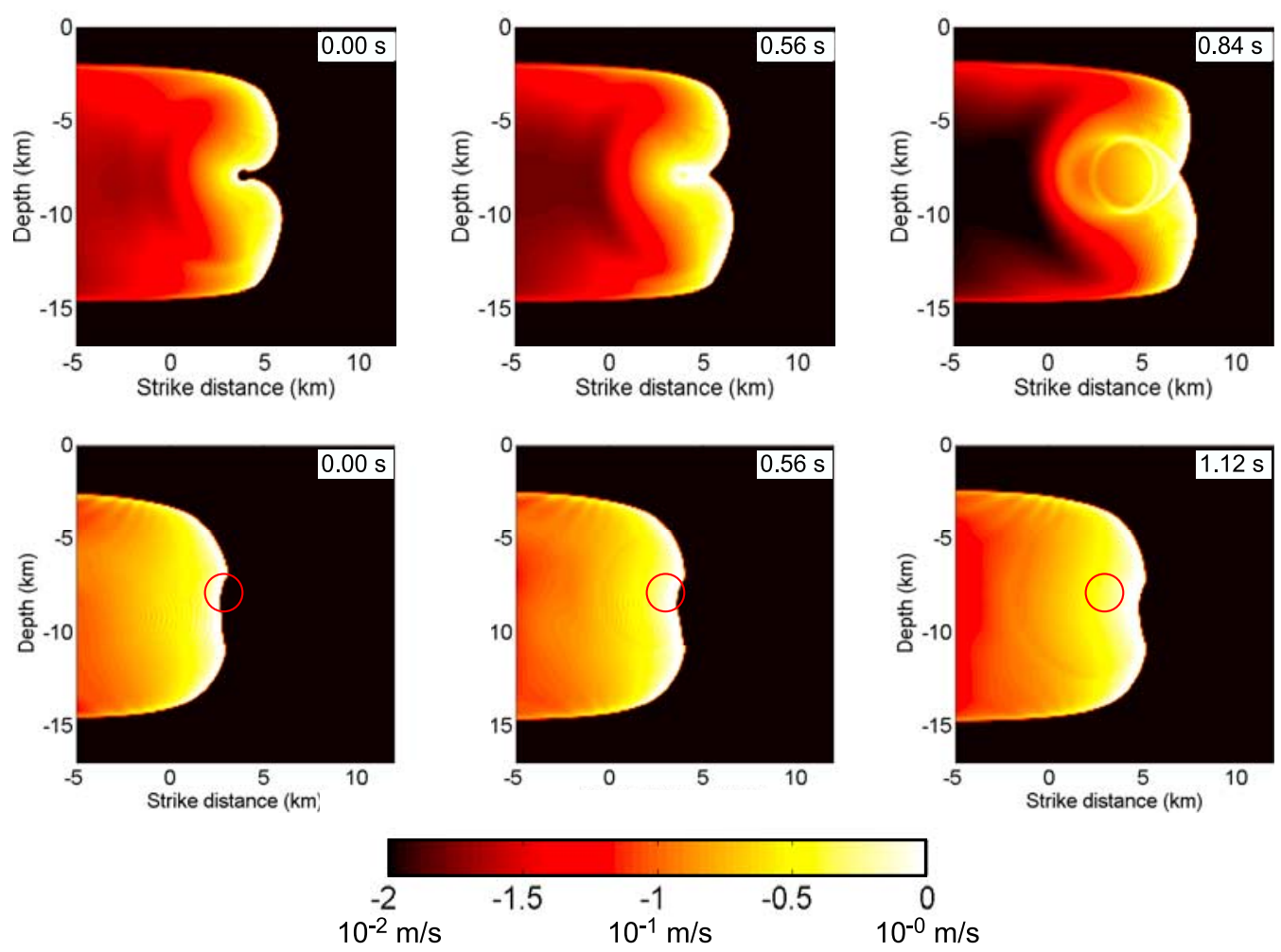

Figure 13. Snapshots of slip velocity distribution during the (top) first and (bottom) second events for the case with a stronger patch. The slip velocity range shown is different from Figures $4-6$ and chosen to emphasize the rupture front. The number in the top right corner of each snapshot indicates the elapsed time (in seconds) since the first snapshot for each event. During the first event, dynamic rupture interacts with the stronger patch and produces a supershear burst. During the second event, no interaction or supershear propagation occurs; the stronger patch is indicated by a red circle in this case. Rupture behavior of the first event does not repeat in the slip history of the fault due to redistribution of shear stress.

the ratio $\tau / \sigma$ is 0.62 inside the patch and 0.63 outside of it. In other words, before the second event, shear stress is proportionally higher at the patch, compensating for its higher strength.

[51] This simple example illustrates the necessity of longterm simulations of fault behavior for understanding effects of fault heterogeneity, even if one is concerned with dynamic events only. While any prestress can be assumed for simulations of a single dynamic rupture, stress distribution before events in long-term simulations as well as on natural faults is the result of complicated history of seismic and aseismic slip, which depends, in part, on the strength distribution. Our results suggest that distributions of fault stress and strength are related and cannot be assumed independently.

\subsection{Effect of Heterogeneity on Long-Term Behavior}

[52] The $20 \%$ stronger patch occupies less that $1 \%$ of the fault area, yet it changes the long-term behavior of slip in this model. We compare the homogeneous fault case of section 3.2 and the case of one small heterogeneity considered here using plots of slip accumulation along the horizontal line $z=-8 \mathrm{~km}$ (Figures $7 \mathrm{a}$ and 15a). We see that while the sequence of large events maintains its two-event periodicity, the stronger patch destroys the symmetry in fault properties between the left and right sides of the fault, resulting in asymmetric behavior. In the homogeneous case, aseismic transients alternate the direction of their propagation, moving from left to right before one event and the other way before the next one. In the case with the stronger patch, all aseismic slip propagates from left to right. Once the slow slip reaches the stronger patch, an event nucleates. This is because larger effective normal stress corresponds to a smaller nucleation size, favoring rupture nucleation there. Correspondingly, the nucleation process at the right side of the fault is modified and shifted to occur at the asperity.

\section{Quasi-Dynamic Approach}

[53] If the fully dynamic formulation is replaced with the quasi-dynamic one [e.g., Rice, 1993], simulations become much simplified and computational resources needed are significantly reduced. The quasi-dynamic formulation has been widely used in earthquake studies [e.g., Rice, 1993; Ben-Zion and Rice, 1995; Rice and Ben-Zion, 1996; Hori et al., 2004; Kato, 2004; Hillers et al., 2006; Ziv and Cochard, 2006]. It ignores wave-mediated stress transfers expressed through convolutions integrals in equation (5) by setting 


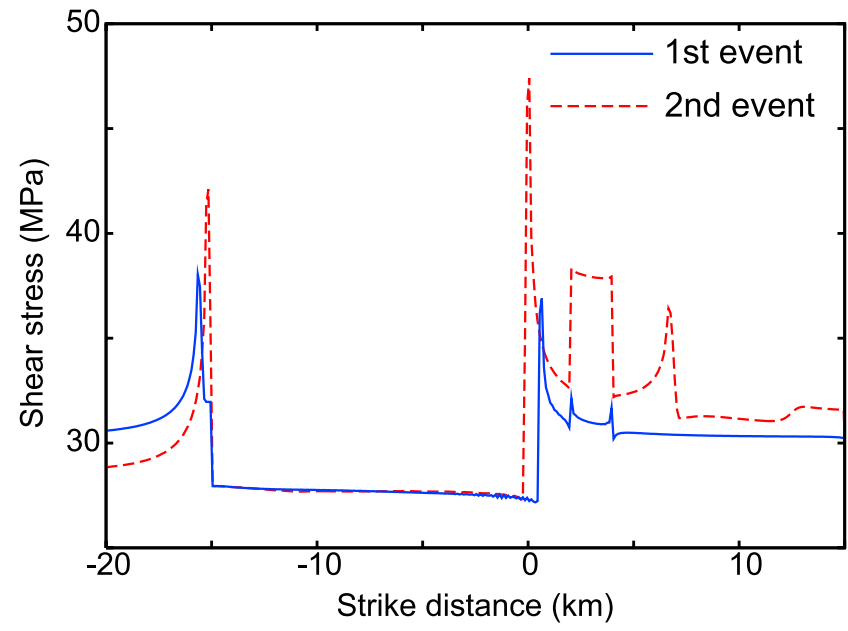

Figure 14. Distribution of shear stress along the line $z=$ $-8 \mathrm{~km}$ during the first and second events, at the time when the horizontal rupture front (at this depth) arrives approximately at the center of the fault $(x=0 \mathrm{~km})$. In the first event, the patch has shear stress $\tau$ similar to the surrounding area but $20 \%$ larger normal stress $\sigma$, resulting in smaller nondimensional prestress $\tau / \sigma$ than the rest of the fault. However, in the second event, $\tau$ inside the patch is about $20 \%$ larger than in the surrounding area, resulting in homogeneous nondimensional prestress $\tau / \sigma$. This redistribution of shear stress due to prior slip history eliminates the interaction of dynamic rupture with the patch observed in the first event.

$T_{w}=0$ for all Fourier modes. Any increment of slip induces instantaneous static stress changes everywhere on the fault. However, the quasi-dynamic formulation differs from the quasi-static one in that it retains dynamic radiation terms $\eta_{i} V_{i}(x, z ; t)$ in equation (1), capturing some dynamic effects and allowing the solution to exist during dynamic instabilities.

[54] Here we compare the results of quasi-dynamic 3-D calculations with the fully dynamic ones and explore the suggestion of Lapusta et al. [2000] that smaller radiation damping terms in the quasi-dynamic formulation can make the comparison more favorable. For this study, we use the model with a stronger patch from section 5 .

\subsection{Generalized Quasi-Dynamic Formulation}

[55] Let us generalize the quasi-dynamic formulation to allow for smaller radiation damping coefficients. Shear components of tractions can be written as

$$
\tau_{i}(x, z ; t)=\tau_{i}^{o}(x, z ; t)+f_{i}(x, z ; t)-\frac{\mu}{2 c_{\mathrm{s}} \beta_{\mathrm{s}}} V_{i}(x, z ; t),
$$

where $i=x, z, \beta_{\mathrm{s}}$ is a constant, and the convolution integrals in $f_{i}(x, z ; t)$ are ignored. For the standard quasi-dynamic formulation, we have $\beta_{\mathrm{s}}=1$, and we are interested in $\beta_{\mathrm{s}} \geq 1$. Wave speeds enter the quasi-static formulation through the radiation damping terms in (24) and through the static stress transfers in (5) which use the ratio $c_{\mathrm{p}} / c_{\mathrm{s}}$. Therefore, the generalized quasi-dynamic formulation (24) with $\beta_{\mathrm{s}}>1$ corresponds to the standard quasi-dynamic approach with faster wave speeds:

$$
\bar{c}_{\mathrm{s}}=\beta_{\mathrm{s}} c_{\mathrm{s}}, \bar{c}_{\mathrm{p}}=\beta_{\mathrm{s}} c_{\mathrm{p}}
$$

Because of the faster wave speeds, we need to use smaller time steps $\overline{\Delta t}_{\text {min }}$ in calculations with $\beta_{\mathrm{s}}>1$ :

$$
\overline{\Delta t}_{\min }=\gamma \frac{\Delta x}{\bar{c}_{\mathrm{s}}}=\gamma \frac{\Delta x}{\beta_{\mathrm{s}} c_{\mathrm{s}}}=\frac{\Delta t_{\mathrm{min}}}{\beta_{s}} .
$$

We have confirmed conclusion (26) in our simulations. This means that simulating quasi-dynamic problems with smaller radiation damping terms is more challenging, as it requires smaller time steps and more computational time. This consideration is also consistent with the fact that the quasistatic formulation cannot be used to model dynamic rupture. For the quasi-static formulation, $\beta_{\mathrm{s}}=+\infty$ and, according to equation (26), the time steps should be infinitely small.

\subsection{Similarity of Quasi-Dynamic Solutions and Their Differences With Fully Dynamic Results}

[56] Let us compare the fully dynamic simulation of section 5 with several quasi-dynamic simulations that use different values of $\beta_{\mathrm{s}}$. Figure 15 shows slip accumulation along the horizontal line $z=-8 \mathrm{~km}$ for the fully dynamic simulation and the quasi-dynamic simulations with $\beta_{\mathrm{s}}=1$, 2 , and 4 . We choose a part of slip history that already reflects the long-term behavior of the model. The first observation is that the rupture speed and slip velocity, which are related to the horizontal and vertical spacing of red dashed lines, respectively, are much lower for the standard quasi-dynamic simulation $\left(\beta_{\mathrm{s}}=1\right)$ than for the fully dynamic one. However, the rupture speed and slip velocity increase for larger $\beta_{\mathrm{s}}$ and, for $\beta_{\mathrm{s}}=4$, look comparable to that of the fully dynamic simulation.

[57] Hence, it is tempting to conclude that larger values of $\beta_{\mathrm{s}}$ result in a better match. However, further examination reveals a problem. All quasi-dynamic simulations share a qualitatively similar one-event slip pattern. The fully dynamic simulation has a different slip pattern that consists of two events, as discussed in section 5.3. Hence, it seems that differences that accumulate during dynamic events are sufficient to change long-term fault behavior even in this relatively simple model.

[58] Comparison of individual events in Figure 16 further demonstrates the similarity among quasi-dynamic simulations and their differences with the fully dynamic one. Slip and slip velocity histories of one point on the fault $(x=9 \mathrm{~km}$ and $z=-8 \mathrm{~km}$ ) during the first event are shown in Figures $16 \mathrm{a}$ and $16 \mathrm{~b}$. Zero for each time history is chosen as the time of rupture arrival at the point with $x=6 \mathrm{~km}$ and $z=$ $-8 \mathrm{~km}$. From Figure 16a, we can get the average rupture speed between locations $x=6 \mathrm{~km}$ and $x=9 \mathrm{~km}$ along the line $z=-8 \mathrm{~km}$, which is $0.96 \mathrm{~km} / \mathrm{s}$ for quasi-dynamic simulation with $\beta_{\mathrm{s}}=1,1.92 \mathrm{~km} / \mathrm{s}$ for $\beta_{\mathrm{s}}=2,3.79 \mathrm{~km} / \mathrm{s}$ for $\beta_{\mathrm{s}}=4$, and $2.65 \mathrm{~km} / \mathrm{s}$ for the fully dynamic simulation. Hence, the rupture speed in the quasi-dynamic simulation with $\beta_{\mathrm{s}}=4$ is faster than the fully dynamic simulation. At the same time, slip velocity for $\beta_{\mathrm{s}}=4$, while substantially higher than that for $\beta_{\mathrm{s}}=1$, is still much smaller than slip 

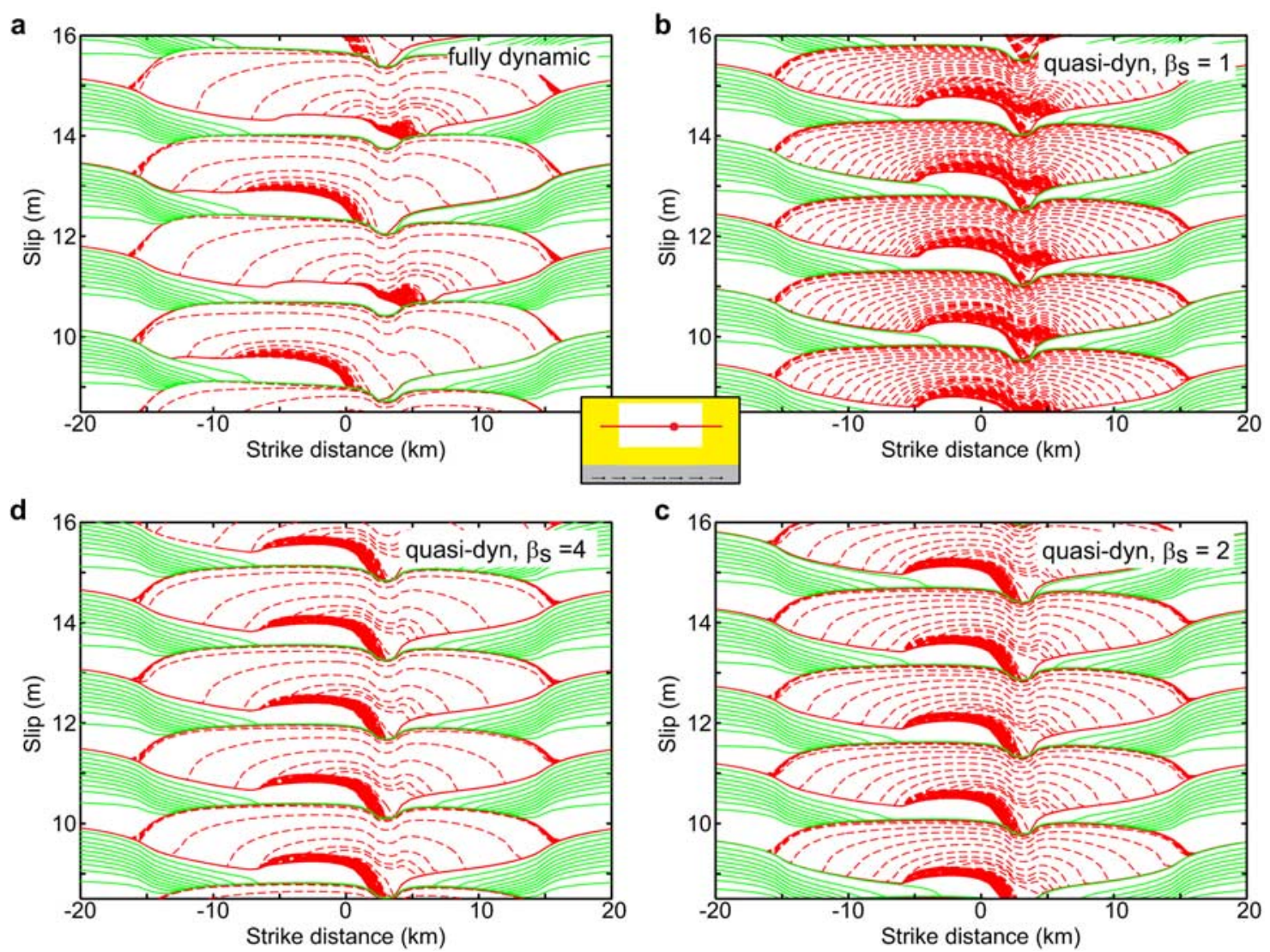

Figure 15. Accumulation of slip along the line $z=-8 \mathrm{~km}$ for the case with a stronger patch. Lines have the same meaning as in Figure 7. (a) Results for the fully dynamic simulation. The slip pattern of the fault with a small stronger patch (which occupies less than 1\% of the seismogenic area) is different from the one with the homogeneous seismogenic region (Figure 7a). (b) The standard quasi-dynamic formulation $\left(\beta_{s}=1\right)$ results in a modified slip pattern, smaller slip velocity, slower rupture speeds, and smaller slip per event. (c and $\mathrm{d}$ ) Larger values of $\beta_{s}$ or smaller radiation terms in the quasi-dynamic formulation accelerate rupture speed and increase slip velocity. However, all quasi-dynamic simulations produce similar slip patterns that are qualitatively different from the fully dynamic one.

velocity of the fully dynamic calculation. Furthermore, the final slip is smaller for all quasi-dynamic simulations (Figure 16b) than for the fully dynamic one; this is also true about average slip per event and static stress drop. Increasing $\beta_{\mathrm{s}}$ further is not a productive approach to increasing slip velocity and slip rates, as the rupture speed would also increase, and the rupture speed for $\beta_{\mathrm{s}}=4$ is already too high, as demonstrated in Figure 16c. For points between $x=6 \mathrm{~km}$ and $x=15 \mathrm{~km}$, the rupture speed of the quasi-dynamic simulation with $\beta_{\mathrm{s}}=4$ is higher than that of the fully dynamic run. Note that the standard quasidynamic approach (with $\beta_{\mathrm{s}}=1$ ) fails to reproduce the supershear burst during the first dynamic event discussed in section 5.1.

[59] We find that quasi-dynamic simulations with different values of $\beta_{\mathrm{s}}$ can be scaled to match each other. The insets in Figures $16 \mathrm{a}$ and $16 \mathrm{~b}$ show rescaled slip velocity $V^{*}$ $=V / \beta_{\mathrm{s}}$ and slip $\delta$ as functions of the rescaled time $t^{*}=\beta_{\mathrm{s}} t$. Figure $16 \mathrm{~d}$ plots rescaled rupture speeds $c /\left(\beta_{\mathrm{s}} c_{\mathrm{s}}\right)$, with the fully dynamic result for comparison. We see that all quasidynamic curves fall almost on top of each other in rescaled plots. Hence, quasi-dynamic simulations with different $\beta_{s}$ are similar to each other during dynamic rupture, provided we use rescaled time $t^{*}=\beta_{s} t$, slip velocity $V^{*}=V / \beta_{s}$, and rupture speed $c^{*}=c / \beta_{s}$ (with stress $\tau$, slip $\delta$, and spatial coordinates $x, z$ unchanged).

\subsection{Cohesive Zone Size and Numerical Resolution in Quasi-Dynamic Simulations}

[60] We find that the cohesive zone size in quasi-dynamic calculations is always equal to the quasi-static cohesive zone size $\Lambda_{0}$. It does not decrease as rupture propagates and it is independent of $\beta_{\mathrm{s}}$. Figure 17 shows stress distribution along parts of the line $z=-8 \mathrm{~km}$ during the first event for the quasi-dynamic simulations with $\beta_{\mathrm{s}}=1$ and $\beta_{\mathrm{s}}=4$. The simulated events are shown in Figure 17 (bottom). Note that we use $\Delta x=50 \mathrm{~m}$ in these simulations, instead of $100 \mathrm{~m}$, to better capture the size of the cohesive zone. In Figure 17a of $\beta_{\mathrm{s}}=1$, the rupture front is at $x=-7.9 \mathrm{~km}$ and the average rupture speed before that location is only $0.12 \mathrm{~km} / \mathrm{s}$, which is less than $5 \%$ of the shear wave speed. The cohesive zone size in that situation, which is equal to 6 cell sizes or $300 \mathrm{~m}$, should be very close to the quasi-static cohesive zone size. The theoretical estimate in section 4.1 gives a similar value of $233 \mathrm{~m}$. At the later stages of this rupture, the cohesive zone size stays equal to $300 \mathrm{~m}$. For the quasi-dynamic 

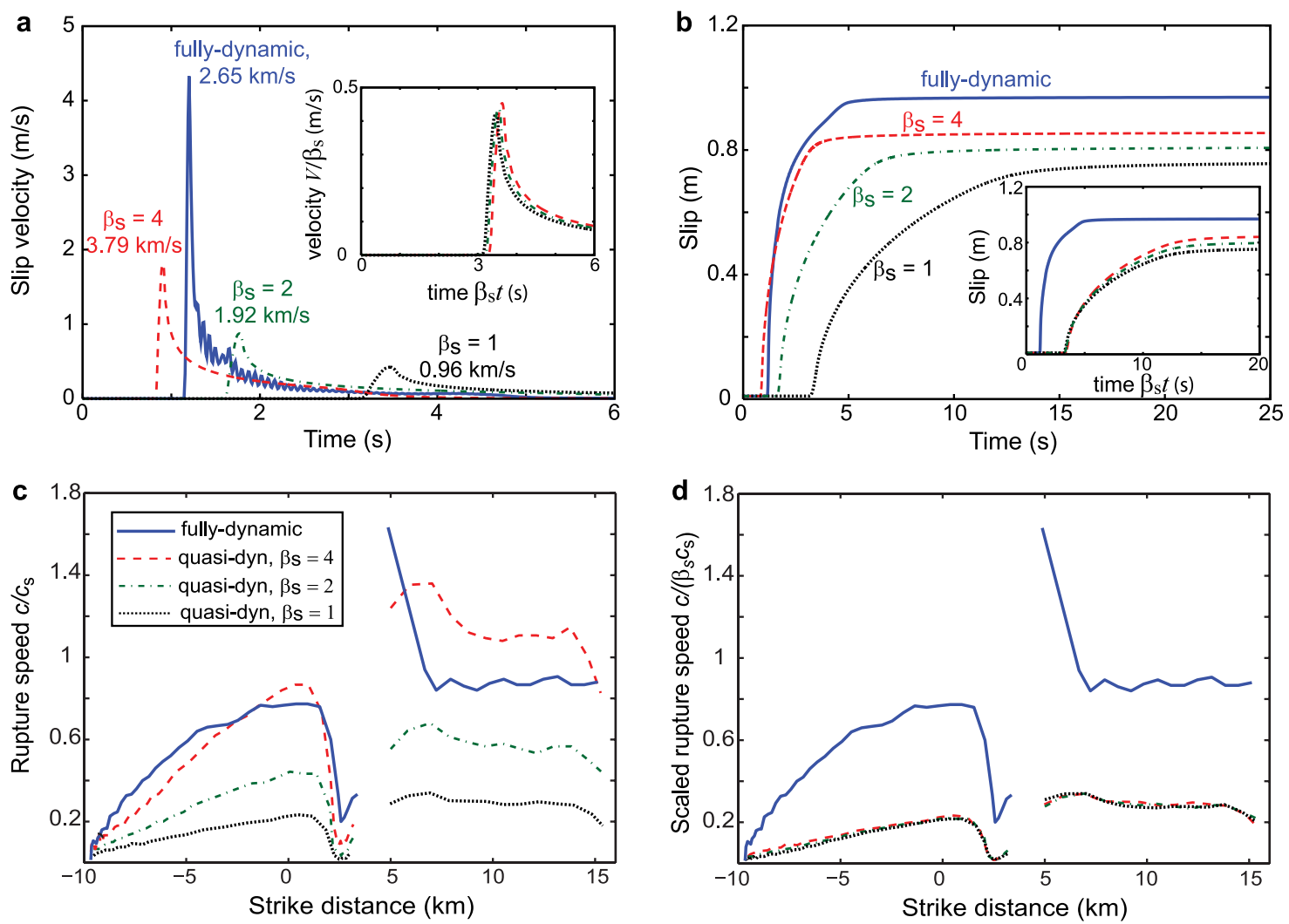

Figure 16. Comparison of fully dynamic and quasi-dynamic simulations of one dynamic event (the first event in the sequence). ( $\mathrm{a}$ and b) Slip velocity and slip histories of the fault location $(9 \mathrm{~km},-8 \mathrm{~km})$. Zero time corresponds to the time of rupture arrival at the point $(6 \mathrm{~km},-8 \mathrm{~km})$. Slip velocity and slip per event in quasi-dynamic simulations are significantly smaller than in the fully dynamic one. Simulations with larger $\beta_{s}$ produce faster rupture speeds, larger slip velocity, and larger slip per event. However, when scaled appropriately, the quasi-dynamic results all collapse onto the same curves (insets in Figures 16a and 16b). (c and d) Rupture speed as a function of rupture tip location along $z=-8 \mathrm{~km}$. The quasidynamic simulation with $\beta_{s}=4$ has larger rupture speeds than the fully dynamic simulation. All quasidynamic simulations have nearly identical scaled rupture speed $c /\left(\beta_{s} c_{\mathrm{s}}\right)$, as shown in Figure $16 \mathrm{~d}$.

simulation with $\beta_{s}=4$, the cohesive zone size is still $300 \mathrm{~m}$ (Figure 17b). At the time shown in Figure 17b, the rupture speed is $2.45 \mathrm{~km} / \mathrm{s}$, more than $80 \%$ of the shear wave speed.

[61] The fact that the cohesive zone does not change in quasi-dynamic simulations simplifies choosing proper spatial discretization. In our study, any cell size below the quasi-static estimate of the cohesive zone size have produced well-resolved quasi-dynamic calculations (we have tried $\Delta x=50 \mathrm{~m}, 100 \mathrm{~m}$, and $200 \mathrm{~m}$; recall that $\Delta x=200 \mathrm{~m}$ was not adequate for the fully dynamic simulation). But larger cell sizes lead to inaccurate solutions; for example, $\Delta x=400 \mathrm{~m}$ changes the results significantly, just like in the fully dynamic case. How much more advantageous is the quasi-dynamic simulation in terms of the spatial discretization depends on how much the cohesive zone shrinks during the corresponding fully dynamic simulation.

\section{Conclusions}

[62] We have developed, on the basis of prior studies, a 3-D methodology for simulating long-term history of spontaneous seismic and aseismic slip on a vertical planar strike- slip fault subjected to slow tectonic loading. Our approach reproduces all stages of earthquake cycles, from accelerating slip before dynamic instability, to rapid dynamic propagation of earthquake rupture, to postseismic slip, and to interseismic creep, including aseismic transients. We have extended the existing 2-D methodology [Lapusta et al., $2000]$ to $3-\mathrm{D}$, proposed a numerical resolution criterion that combines findings for long-term histories and dynamic rupture, developed a new frequency-dependent truncation procedure, determined the values of numerical parameters that lead to results independent of numerical procedures in three dimensions, developed a parallel implementation of the 3-D code, and applied the developed methodology to several examples.

[63] In 2-D studies of earthquake sequences [e.g., Rice, 1993; Lapusta et al., 2000], the numerical discretization was based on the need to resolve the nucleation process and the associated spatial scale $h^{*}$. However, in both fully dynamic and quasi-dynamic simulations, seismic events propagate as dynamic ruptures with rapid variations of field variables at their tip. We have shown that the resolution criterion based on the near tip cohesive zone and quantified 
a
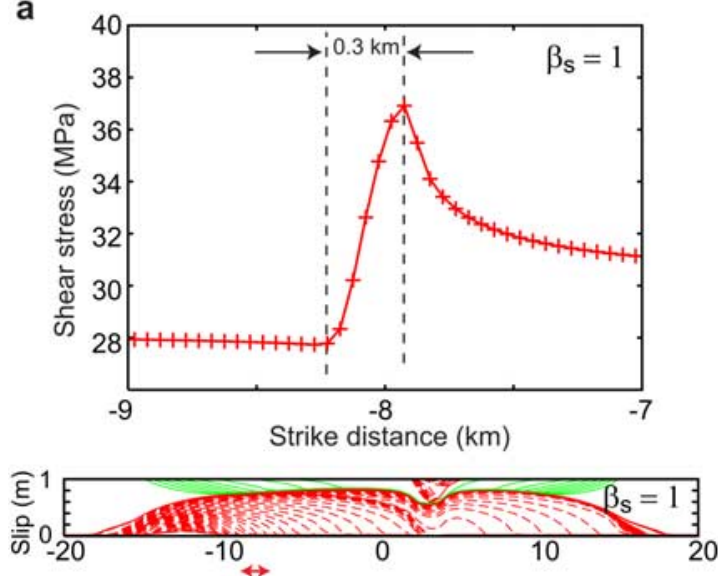

b

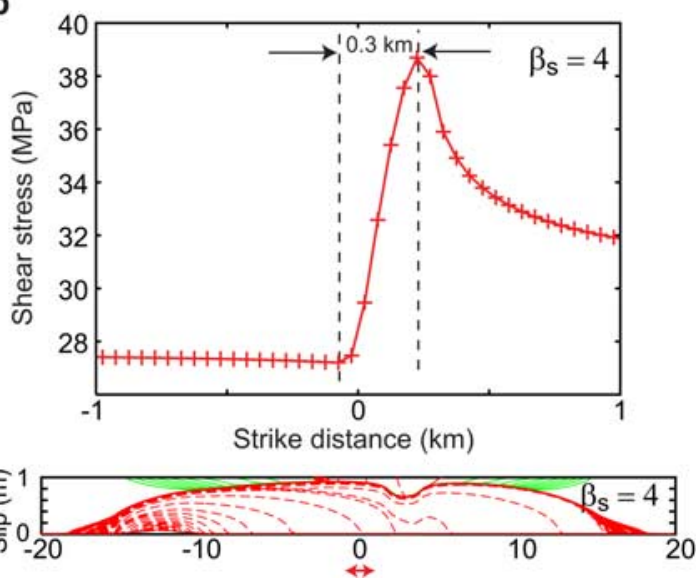

Figure 17. Cohesive zones in quasi-dynamic simulations. (a) (top) Shear stress distribution along the horizontal line $z=-8 \mathrm{~km}$ at the time of rupture front arrival at point $(-7.9 \mathrm{~km},-8 \mathrm{~km})$ during the first event in the simulation with $\beta_{s}=1$. Crosses indicate locations of spatial cells $(\Delta x=50 \mathrm{~m})$. The rupture speed at that time is $0.12 \mathrm{~km} / \mathrm{s}$. The cohesive zone size is $0.3 \mathrm{~km}$. (bottom) Accumulation of slip in that case, with the double arrow indicating the distance plotted in Figure 17a (top). (b) Shear stress distribution along the horizontal line $z=-8 \mathrm{~km}$ at the time of rupture front arrival at $(0.24 \mathrm{~km},-8 \mathrm{~km})$ $\mathrm{km}$ during the first event in the simulation with $\beta_{s}=4$. The rupture speed at that time is $2.45 \mathrm{~km} / \mathrm{s}$. Despite the different value of $\beta_{s}$ and the different rupture speed, the cohesive zone size is still $0.3 \mathrm{~km}$. In quasi-dynamic simulations, the cohesive zone size does not shrink during rupture propagation and its size is independent of the parameter $\beta_{s}$.

in our previous collaborative work [Day et al., 2005] is more restrictive for the parameters typically used in earthquake sequence simulations. Once the cohesive zone size is resolved with several spatial cells, the nucleation-related scale $h^{*}$ is resolved as well, since it is several time larger. This consideration explains the finding of Lapusta et al. [2000] that $h^{*}$ needs to be resolved by 20 spatial cells, a relatively large number; that level of discretization was actually required for resolving the much smaller size of the cohesive zone.

[64] We find that the frequency-dependent truncation procedure developed for 2-D antiplane problems by Lapusta et al. [2000] is inadequate in three dimensions. This is because 3-D problems involve elastodynamic kernels for mode II, and those kernels decay much slower than the mode III kernels. We have developed a new frequencydependent truncation procedure that is based both on considerations of Lapusta et al. [2000] and on the decay of the kernel amplitude.

[65] Slip response in the presented fault models, which have relatively simple distributions of friction properties, involves aseismic transients, i.e., episodes of spontaneous aseismic fault slip faster than the plate rate. These transients arise in the areas of velocity-weakening regions close to rheological transitions and constitute propagating nucleation attempts. Their extent depends on the nucleation size. In our simulations, we choose parameters that make nucleation sizes a significant fraction of the fault width, to make the problems numerically tractable. Large nucleation sizes may be realistic for certain fault conditions such as highly elevated pore pressure or velocity-weakening properties close to velocity-neutral. In fact, Liu and Rice [2005] obtained aseismic transients in a subduction model with occasional highly elevated pore pressure next to rheological transition. It is possible that aseismic transients occur only under conditions that result in large nucleation sizes, in which case the mechanism of aseismic transients presented by Liu and Rice [2005] and reproduced here is a viable one. However, note that many areas on natural faults should have small nucleation sizes to produce small events. Other mechanisms have been proposed to explain aseismic transients, such as inelastic dilatancy and complex dependence of friction on slip velocity [e.g., Segall and Rubin, 2007; Shibazaki and Shimamoto, 2007].

[66] We have used the developed methodology to investigate interaction of slip with a stronger fault patch of $20 \%$ higher normal stress over many earthquake cycles. The patch significantly affects the dynamic rupture in the first event, causing rupture delay followed by a supershear burst. However, the patch becomes "invisible" to dynamic rupture in subsequent events due to redistribution of shear stress. Our results show that while simulations of single dynamic events play an important role in exploring earthquake dynamics, long-term simulations are also important as they can help us understand how assumptions about the distribution of fault strength influence the distribution of fault stress before large events. Our results have consequences for studies that attempt to determine parameters of strong ground motion by considering many potential scenarios of earthquake rupture on a given fault, primarily by choosing different distributions of fault strength and fault initial conditions. Our results suggest that the two distributions are related owing to prior fault slip. Note that the small stronger patch, which occupies only $1 \%$ of the fault areas, significantly influences the long-term behavior of the fault 
in our model, moving nucleation locations and causing asymmetric behavior.

[67] Comparison of the fully dynamic and the standard quasi-dynamic approaches shows that the quasi-dynamic approach results in smaller slip per event and significantly smaller slip velocities and rupture speeds, confirming the results of 2-D comparisons [Lapusta et al., 2000]. The new observation in 3-D models is that the long-term slip pattern of the model is also different between the fully dynamic and quasi-dynamic simulations, even for the simple distributions of fault properties considered. Hence, the discrepancies between the fully dynamic and quasi-dynamic simulations accumulated during inertially controlled ruptures have longterm influences over earthquake cycles in three dimensions. The quasi-dynamic approach also fails to reproduce the supershear burst in the first dynamic event of the simulation with a stronger patch. We have explored the possibility of improving the comparison by decreasing radiation damping terms of the quasi-dynamic formulation. We find that such a change is equivalent to the standard formulation with higher wave speeds and it only rescales the resulting solution without changing it qualitatively.

[68] The developed methodology can be used to study a number of fault slip phenomena, such as postseismic slip, earthquake nucleation in heterogeneous fault regions, slip response of faults with complex patterns of velocity-weakening and velocity-strengthening properties, and interaction of fault slip with heterogeneity in normal stress or other fault properties. Application of this approach to small repeating earthquakes explained the scaling of their seismic moment with recurrence time [Chen and Lapusta, 2009]. The methodology can be easily adapted to rate and state formulations other than the aging formulation considered in this work, and to combinations of rate and state friction with dynamic weakening mechanisms. Recent improvements in availability and quality of seismic and geodetic data have revealed complex interactions of seismic and aseismic slip, with more heterogeneous fault coupling than previously where

$$
\begin{gathered}
C_{\mathrm{II}}=\frac{J_{1}(\rho)}{\rho}+4 \rho\left[W\left(\frac{c_{\mathrm{p}}}{c_{\mathrm{s}}} \rho\right)-W(\rho)\right]-4 \frac{c_{\mathrm{s}}}{c_{\mathrm{p}}} J_{0}\left(\frac{c_{\mathrm{p}}}{c_{\mathrm{s}}} \rho\right)+3 J_{0}(\rho), \\
C_{\mathrm{III}}=\frac{J_{1}(\rho)}{\rho}, \\
W(\rho)=\int_{\rho}^{\infty} \frac{J_{1}(\eta)}{\eta} d \eta=1-\int_{0}^{\rho} \frac{J_{1}(\eta)}{\eta} d \eta,
\end{gathered}
$$

and $J_{0}(\rho)$ and $J_{1}(\rho)$ denote Bessel functions. Kernels $K_{\mathrm{II}}(\rho)$ and $K_{\mathrm{III}}(\rho)$ have the following property:

$$
\int_{0}^{\infty} K_{\mathrm{II}}(\eta) d \eta=\int_{0}^{\infty} K_{\mathrm{III}}(\eta) d \eta=1
$$

The asymptotic form of Bessel functions $J_{n}(\rho)$ for $\rho \gg n$ is

$$
J_{n}(\rho) \sim \sqrt{\frac{2}{\pi \rho}} \cos \left(\rho-\frac{1}{2} n \pi-\frac{1}{4} \pi\right)
$$

and $K_{\mathrm{III}}(\rho)$ can be expanded for $\rho \gg 1$ :

$$
\begin{aligned}
K_{\mathrm{III}}(\rho) & =\int_{\rho}^{\infty} \frac{J_{1}(\eta)}{\eta} d \eta \\
& \sim \sqrt{\frac{2}{\pi}}\left\{\frac{\cos (\rho-\pi / 4)}{\rho^{\frac{3}{2}}}+\frac{3}{2} \frac{\sin (\rho-\pi / 4)}{\rho^{\frac{5}{2}}}+O\left(\rho^{-\frac{7}{2}}\right)\right\} .
\end{aligned}
$$

The leading term of the expansion is given by

$$
K_{\mathrm{III}}^{\mathrm{app}}(\rho)=\sqrt{\frac{2}{\pi}} \frac{\cos (\rho-\pi / 4)}{\rho^{\frac{3}{2}}} .
$$

Similarly, the asymptotic expansion of $K_{\mathrm{II}}(\rho)$ for $\rho \gg 1$ is

$$
K_{\mathrm{II}}(\rho)=\sqrt{\frac{2}{\pi}}\left\{\frac{\sin (\rho-\pi / 4)}{\rho^{\frac{1}{2}}}+\frac{4\left(c_{\mathrm{s}} / c_{\mathrm{p}}\right)^{\frac{7}{2}} \cos \left(c_{\mathrm{p}} \rho / c_{\mathrm{s}}-\pi / 4\right)-3 \cos (\rho-\pi / 4)}{\rho^{\frac{3}{2}}}+O\left(\frac{1}{\rho^{\frac{5}{2}}}\right)\right\}
$$

believed, slow earthquakes, and aseismic transients that are often accompanied by seismic tremor. Interpreting this rich information through forward modeling developed in this work can help us determine the constitutive behavior and properties of natural faults.

\section{Appendix A: Convolution Kernels}

[69] The elastodynamic convolution kernels $K_{\mathrm{II}}$ and $K_{\mathrm{III}}$ from equation (5) can be expressed as [Geubelle and Rice, 1995]

$$
\begin{gathered}
K_{\mathrm{II}}(\rho)=2\left(1-c_{\mathrm{s}}^{2} / c_{\mathrm{p}}^{2}\right)-\int_{0}^{\rho} C_{\mathrm{II}}(\eta) d \eta \\
K_{\mathrm{III}}(\rho)=1-\int_{0}^{\rho} C_{\mathrm{III}}(\eta) d \eta
\end{gathered}
$$

with the leading term

$$
K_{\mathrm{II}}^{\mathrm{app}}(\rho)=\sqrt{\frac{2}{\pi}} \frac{\sin (\rho-\pi / 4)}{\rho^{\frac{1}{2}}} .
$$

The kernels and their comparison with the leading terms are shown in Figure A1. The slower decay of $K_{\mathrm{II}}(\rho)$ has important implications for the frequency-dependent truncation, as discussed in sections 2.2 and 4.3.

[70] In our simulations, slip velocity histories exhibit some small oscillations (e.g., Figure 12). For both $\Delta x=$ $100 \mathrm{~m}$ and $\Delta x=50 \mathrm{~m}$, the oscillations have the period of 6 time steps, or $0.0672 \mathrm{~s}$ for $\Delta x=100 \mathrm{~m}$ and $0.0336 \mathrm{~s}$ for $\Delta x=50 \mathrm{~m}$. The amplitude of the oscillations gets smaller for $\Delta x=50 \mathrm{~m}$. Hence, the oscillations are not physical. We find that they are due to kernel discretization. As shown above, kernels $K_{\mathrm{II}}(\rho)$ and $K_{\mathrm{III}}(\rho)$ have the period of $2 \pi$ for $\rho \gg 1$. In simulations, time $t$ is discretized with the 
a
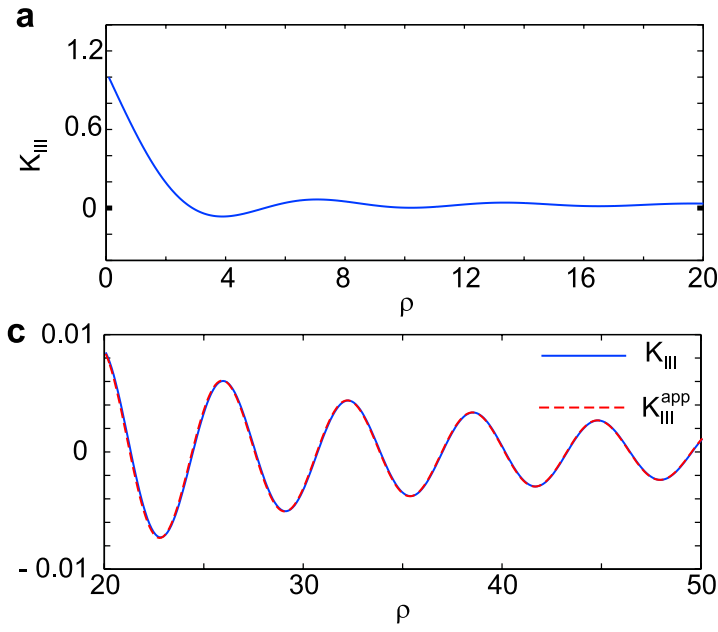

b

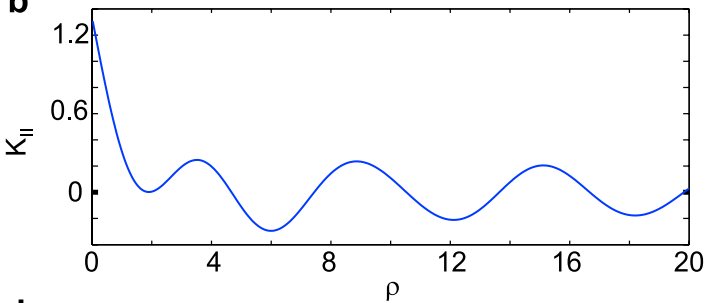

d 0.06

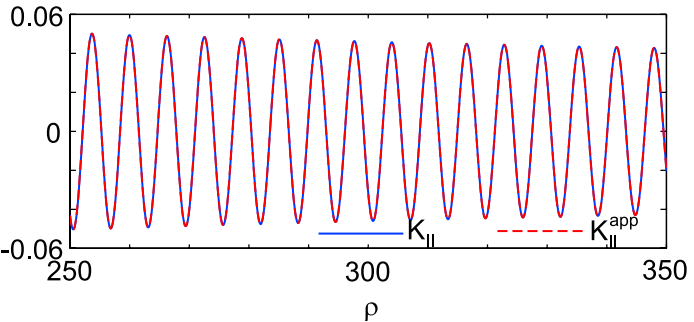

Figure A1. Elastodynamic kernels $K_{\mathrm{III}}(\rho)$ and $K_{\mathrm{II}}(\rho)$. (a and b) Values of the kernels for relatively small kernel arguments. (c and d) Comparison of kernels with the leading terms in their asymptotic expansions. For $\rho \gg 1, K_{\mathrm{III}}(\rho) \sim O\left(\rho^{-3 / 2}\right)$ and $K_{\mathrm{II}}(\rho) \sim O\left(\rho^{-1 / 2}\right) . K_{\mathrm{II}}(\rho)$ decays much slower than $K_{\text {III }}(\rho)$ as $\rho$ increases.

minimum time step $\Delta t_{\min }=\gamma \Delta x / c_{\mathrm{s}}$ and hence the kernel argument $\rho$ is discretized with the kernel interval given by

$$
\Delta \rho_{\min }=\hat{q} c_{\mathrm{s}} \Delta t_{\min }
$$

Therefore, for each frequency mode $\hat{q}$, the number of points resolving one period of the kernel is

$$
N_{\rho}=\frac{2 \pi}{\Delta \rho_{\min }}=\frac{2 \pi}{\hat{q} \gamma \Delta x} .
$$

$N_{\rho}$ is smaller for larger $\hat{q}$.

[71] For the highest-frequency magnitude $\hat{q}_{\max }=\sqrt{2} \pi /$ $\Delta x$, we get $N_{\rho}=\sqrt{2} / \gamma=4.2$ for $\gamma=1 / 3$ that we use in simulations. Therefore, one period of the kernel is resolved by only 4.2 points for the highest frequency. However, only four modes $\left(k= \pm N_{x} / 2, m= \pm N_{z} / 2\right)$ have such high frequency. If one considers all the modes as a rectangular array, $-\pi / \Delta x \leq \hat{k}=2 \pi k / \lambda_{x} \leq \pi / \Delta x$ and $-\pi / \Delta x \leq \hat{m}=2 \pi z /$ $\lambda_{z} \leq \pi / \Delta x$ with $\hat{q}=\hat{k}^{2}+\hat{m}^{2}$, and draws a circular ring of unit width centered at zero frequency, then the radius of the ring that covers the largest area is $\pi / \Delta x$. Hence, the largest contribution to oscillations likely comes from modes with the frequency magnitude $\hat{q}=\pi / \Delta x$. For these modes, $N_{\rho}=$ $2 / \gamma=6$, which explains the observed period of the oscillations. Smaller cell sizes $\Delta x$ help reduce the amplitude of oscillations, as frequencies $\pi / \Delta x$ become much higher for smaller $\Delta x$, and hence, they have smaller Fourier coefficients $\dot{D}_{x}\left(k, m ; t^{\prime}\right)$ and $\dot{D}_{z}\left(k, m ; t^{\prime}\right)$.

\section{Appendix B: Updating Field Variables}

[72] Modeling of long deformation histories with periods of fast slip requires variable time stepping. We employ the time stepping scheme developed by Lapusta et al. [2000] for 2-D antiplane problems. The scheme works quite well in our 3-D models. The variable time step $\Delta t$ is chosen as

$$
\Delta t=\max \left\{\Delta t_{\min }, \Delta t_{\mathrm{evol}}\right\}
$$

where $\Delta t_{\min }$ is the minimum time step chosen for good resolution of dynamic rupture propagation and $\Delta t_{\text {evol }}$ varies with slip velocity as discussed below. The value of $\Delta t$ is always adjusted to be an integer multiple of $\Delta t_{\min }$, as this simplifies computation of convolution integrals. The minimum time step is given by

$$
\Delta t_{\min }=\gamma \Delta x / c_{\mathrm{s}}
$$

where $\gamma$ is a constant. We use $\gamma=1 / 3$, as this is the value suggested by our previous study of dynamic rupture in three dimensions [Day et al., 2005]. The time step $\Delta t_{\mathrm{evol}}$ is set to be inversely proportional to slip velocity:

$$
\Delta t_{\mathrm{evol}}=\min _{i, j}[\xi(i, j) L(i, j) / V(i, j)],
$$

where $L(i, j), V(i, j)$, and $\xi(i, j)$ are the characteristic slip, slip velocity, and a prescribed parameter for the cell $(i, j)$, $i=1,2, . ., N_{x}$ and $j=1,2, . ., N_{z} . \xi(i, j)$ is a function of friction properties obtained from linear stability analysis [Lapusta et al., 2000]. In addition, $\xi(i, j)$ is constrained to satisfy $\xi(i, j) \leq \xi_{c}$, where $\xi_{c}$ is a constant, to ensure that slip at each time step does not exceed $\xi_{c} L(i, j)$. Lapusta et al. [2000] used $\xi_{c}=1 / 2$. In our 3-D models, we do a series of comparison with $\xi_{c}=1 / 2,1 / 3,1 / 4$, and $1 / 5$. We find that values $\xi_{c} \leq 1 / 3$ produce virtually indistinguishable results, but $\xi_{c}=1 / 2$ results in small differences. In the simulations presented in this work, we use $\xi_{c}=1 / 5$; however, $\xi_{c}=1 / 3$ would have produced the same results.

[73] To update field variables, we extend to 3-D the scheme developed by Lapusta et al. [2000] for 2-D antiplane problems. Instead of updating state variable $\theta$ directly, we use the quantity $\phi=\log \left(V_{o} \theta / L\right)$. From equation (12), the evolution equation for $\phi$ is

$$
\frac{d \phi}{d t}=\frac{V_{o}}{L} e^{-\phi}-\frac{V}{L}
$$


[74] Suppose that at time $t$, the discretized values of tangential slips $\delta_{\nu}(i, j ; t)$, slip velocities $V_{\nu}(i, j ; t), \nu=x, z$ and state variable $\phi(i, j ; t)$ are known for the cell $(i, j), i=1$, $2, . ., N_{x}, j=1,2, . ., N_{z}$. In addition, we also have the Fourier coefficients of tangential slips $D_{\nu}\left(k, m ; t^{\prime}\right)$, and the history of Fourier coefficients of slip velocity $\dot{D}_{\nu}\left(k, m ; t^{\prime}\right),|k| \leq N_{x} / 2$, $|m| \leq N_{z} / 2$ for the (discretized) prior time $t^{\prime}, t-T_{w}<t^{\prime}<t$, where $t$ is the current time and $T_{w}$ is the truncation time window. To advance the field variables by one time step $\Delta t$ and determine the quantities at the time $t+\Delta t$, we proceed in the spirit of a second-order Runge-Kutta procedure as follows (for compactness, all explicit references to the indices $(i, j)$ for physical space and $(k, m)$ for Fourier space are suppressed):

[75] 1. Determine the evolution time step $\Delta t$ using equation (B1).

[76] 2. Make the first predictions of the values of the slips $\delta_{\nu}^{*}(t+\Delta t)$, their Fourier coefficients $D_{\nu}^{*}(t+\Delta t)$ and the state variable $\phi^{*}(t+\Delta t)$ for each cell, assuming that slip velocities are constant and equal to $V_{\nu}(t)$ throughout the time step $t$. Hence, we have

$$
\begin{gathered}
\delta_{\nu}^{*}(t+\Delta t)=\delta_{\nu}(t)+\Delta t V_{\nu}(t), \\
D_{\nu}^{*}(t+\Delta t)=D_{\nu}(t)+\Delta t \dot{D}_{\nu}(t),
\end{gathered}
$$

$$
\phi^{*}(t+\Delta t)= \begin{cases}\log \left\{e^{\phi(t)}(1-V \Delta t / L)+V_{0} \Delta t / L\right\}, & V \Delta t / L \leq 10^{-6} \\ \log \left\{V_{0} / V+\left(e^{\phi(t)}-V o / V\right) e^{-V \Delta t / L}\right\}, & V \Delta t / L>10^{-6}\end{cases}
$$

where $V=\sqrt{V_{x}^{2}(t)+V_{z}^{2}(t)}$ is the resultant slip rate. The update of $\phi$ distinguishes between small and large values of $V \Delta t / L$ for the following reason. For small $V,\left(e^{\phi(t)}-\right.$ $V o / V) \doteq-V o / V$ and $e^{-V \Delta t / L} \doteq 1$ in the numerical sense, and then $\phi^{*}(t+\Delta t) \doteq \log (0)$, which leads to an error in computation. Using the Taylor expansions for small values of $V \Delta t / L$ avoids the problem. Then we compute the first predictions of the Fourier coefficients of stress transfer functionals $F_{\nu}^{*}(t+\Delta t)$, using equation (5):

$$
\begin{aligned}
\left\{\begin{array}{l}
F_{x}^{*}(t+\Delta t) \\
F_{z}^{*}(t+\Delta t)
\end{array}\right\}= & -\frac{\mu}{2 \hat{q}}\left[\begin{array}{cc}
\hat{k}^{2} & \hat{m} \hat{k} \\
\hat{m} \hat{k} & \hat{m}^{2}
\end{array}\right]\left\{2\left(1-\frac{c_{\mathrm{s}}^{2}}{c_{\mathrm{p}}^{2}}\right)\left(\begin{array}{c}
D_{x}^{*}(t+\Delta t) \\
D_{z}^{*}(t+\Delta t)
\end{array}\right)\right. \\
& -\int_{t+\Delta t-T_{w}}^{t} K_{\mathrm{II}}\left(\hat{q} c_{\mathrm{s}}\left(t+\Delta t-t^{\prime}\right)\right)\left(\begin{array}{c}
\dot{D}_{x}\left(t^{\prime}\right) \\
\dot{D}_{z}\left(t^{\prime}\right)
\end{array}\right) d t^{\prime} \\
& \left.-\int_{0}^{\Delta t} K_{\mathrm{II}}\left(\hat{q} c_{\mathrm{s}} t^{\prime}\right) d t^{\prime}\left(\begin{array}{c}
\dot{D}_{x}(t) \\
\dot{D}_{z}(t)
\end{array}\right)\right\}
\end{aligned}
$$$$
+ \text { similarly rewritten second term of (5).(B8) }
$$

For $\Delta t \geq T_{w}$, the second term on the right hand side of the above expression is set to be zero. For $\Delta t<T_{w}$, the term

$f_{\nu}^{*}(t+\Delta t)$ through the inverse fast Fourier transform (FFT) of $F_{\nu}^{*}(t+\Delta t)$.

[77] 3. Find the prediction of slip velocities $V_{\nu}^{*}(t+\Delta t)$ corresponding to the predicted values of the state variable $\phi^{*}(t+\Delta t)$ and stress functionals $f_{\nu}^{*}(t+\Delta t)$. The direction of the slip velocity vector $\boldsymbol{V}=\left(V_{x}, V_{z}\right)$ should coincide with the direction of the shear traction vector $\boldsymbol{\tau}=\left(\tau_{x}, \tau_{z}\right)$ :

$$
\frac{V_{z}^{*}(t+\Delta t)}{V_{x}^{*}(t+\Delta t)}=\frac{\tau_{z}^{*}(t+\Delta t)}{\tau_{x}^{*}(t+\Delta t)} .
$$

Combining (B9) with (1), we get

$$
\begin{gathered}
\frac{V_{z}^{*}(t+\Delta t)}{V_{x}^{*}(t+\Delta t)}=\frac{\Phi_{z}^{*}(t+\Delta t)}{\Phi_{x}^{*}(t+\Delta t)}, \\
\tau^{*}=\Phi^{*}-\frac{\mu}{2 c_{\mathrm{s}}} V^{*},
\end{gathered}
$$

where $\Phi_{\nu}^{*}(t+\Delta t)=\tau_{\nu}^{o}(t+\Delta t)+f_{\nu}^{*}(t+\Delta t), \Phi^{*}=$ $\sqrt{\Phi_{x}^{* 2}(t+\Delta t)+\Phi_{z}^{* 2}(t+\Delta t)}, \quad V^{*}=\sqrt{V_{x}^{* 2}(t+\Delta t)+V_{z}^{* 2}(t+\Delta t)}$, and $\tau^{*}=\sqrt{\tau_{x}^{* 2}(t+\Delta t)+\tau_{z}^{* 2}(t+\Delta t)}$. Equating the shear

traction (B11) to the strength given by the regularized form of the rate and state friction law [e.g., Rice and Ben-Zion, 1996; Ben-Zion and Rice, 1997; Lapusta et al., 2000; Lapusta and Rice, 2003] results in

$$
\Phi^{*}-\frac{\mu}{2 c_{\mathrm{s}}} V^{*}=a \sigma \operatorname{arcsinh}\left[\frac{V^{*}}{2 V_{o}} \exp \left(\frac{f_{o}+b \phi^{*}(t+\Delta t)}{a}\right)\right] .
$$

Newton-Rhapson search is used to solve equation (B12) for $V^{*}$. Once $V^{*}$ are obtained, $V_{\nu}^{*}(t+\Delta t), \nu=x, z$ can be readily found from equation (B10).

[78] 4. Make the second predictions of the values of the slips $\delta_{\nu}^{* *}(t+\Delta t)$, their Fourier coefficients $D_{\nu}^{* *}(t+\Delta t)$, and the state variable $\phi^{* *}(t+\Delta t)$ :

$$
\delta_{\nu}^{* *}(t+\Delta t)=\delta_{\nu}(t)+\Delta t\left[V_{\nu}(t)+V_{\nu}^{*}(t+\Delta t)\right] / 2,
$$

$$
D_{\nu}^{* *}(t+\Delta t)=D_{\nu}(t)+\Delta t\left[\dot{D}_{\nu}(t)+\dot{D}_{\nu}^{*}(t+\Delta t)\right] / 2,
$$

$$
\phi^{* *}(t+\Delta t)= \begin{cases}\log \left\{e^{\phi(t)}\left(1-V^{*} \Delta t / L\right)+V_{0} \Delta t / L\right\}, & V^{*} \Delta t / L \leq 10^{-6}, \\ \log \left\{V_{0} / V^{*}+\left(e^{\phi(t)}-V o / V^{*}\right) e^{-V^{*} \Delta t / L}\right\}, & V^{*} \Delta t / L>10^{-6},\end{cases}
$$

where $\dot{D}^{*}{ }_{\nu}(t+\Delta t)$ are Fourier coefficients of $V^{*}{ }_{\nu}(t+\Delta t)$,

can be computed because slip velocity history is known. The third term is an approximation of the convolution on the time interval corresponding to the current time step. We then obtain the first prediction of stress functionals $\nu=x, z$. Then we can find the corresponding prediction of stress transfer functionals, $f_{\nu}^{* *}(t+\Delta t)$, using $\delta_{\nu}^{* *}(t+\Delta t)$ and assuming the slip velocities are constant and equal to $\left[V_{\nu}(t)+V_{\nu}^{*}(t+\Delta t)\right] / 2$ throughout the evolution time step. 
The computational procedure is analogous to (B8) in stage 2. Note that the second term on the right-hand side of (B8) stays the same in this stage, and hence, we do not need to compute it again.

[79] 6. Find the second prediction of slip velocities $V_{\nu}^{* *}(t+\Delta t)$ that corresponds to the predicted state variables $\phi^{* *}(t+\Delta t)$ and stress functionals $f_{\nu}^{* *}(t+\Delta t)$. The computational procedure is analogous to stage 3 .

[80] 7. Declare the values of the second prediction as the values of field quantities at the time $t+\Delta t$. Store the values of slips $\delta_{\nu}(t+\Delta t)$, their Fourier coefficients $D_{\nu}(t+$ $\Delta t)$, slip velocities $\dot{\delta}_{\nu}(t+\Delta t)$, and state variable $\phi(t+\Delta t)$ for use in the next time step. In addition, store $\dot{D}_{\nu}\left(t^{\prime}\right)=$ $\left[\dot{D}_{\nu}(t)+\dot{D}_{\nu}^{*}(t+\Delta t)\right] / 2, \nu=x, z$ as the Fourier coefficients of slip velocity history for time $t<t^{\prime}<t+\Delta t$. Go back to stage 1 to advance another time step.

[81] Acknowledgments. This study was supported by the National Science Foundation (grant EAR 0548277), the U.S. Geological Survey (grant 07HQGR0070), and the Southern California Earthquake Center (SCEC). SCEC is funded by NSF Cooperative Agreement EAR-0106924 and USGS Cooperative Agreement 02HQAG0008. This is SCEC contribution 1254. The numerical simulations for this research were performed on Caltech Division of Geological and Planetary Sciences Dell cluster. We thank Yoshi Kaneko for discussions.

\section{References}

Bayart, E., A. M. Rubin, and C. Marone (2006), Evolution of fault friction following large velocity jumps, Eos Trans. $A G U, 87(52)$, Fall Meet. Suppl., Abstract S31A-0180.

Ben-Zion, Y., and J. R. Rice (1995), Slip patterns and earthquake populations along different classes of faults in elastic solids, J. Geophys. Res., 100, 12,959-12,983, doi:10.1029/94JB03037.

Ben-Zion, Y., and J. R. Rice (1997), Dynamic simulations of slip on a smooth fault in an elastic solid, J. Geophys. Res., 102, 17,771-17,784, doi:10.1029/97JB01341.

Chen, T., and N. Lapusta (2009), Scaling of small repeating earthquakes explained by interaction of seismic and aseismic slip in a rate and state fault model, J. Geophys. Res., 114, B01311, doi:10.1029/2008JB005749.

Cocco, M., and A. Bizzarri (2002), On the slip-weakening behavior of rateand state dependent constitutive laws, Geophys. Res. Lett., 29(11), 1516, doi:10.1029/2001GL013999.

Cochard, A., and R. Madariaga (1994), Dynamic faulting under rate-dependence friction, Pure Appl. Geophys., 142, 419-445, doi:10.1007/ BF00876049.

Cochard, A., and R. Madariaga (1996), Complexity of seismicity due to highly rate dependent friction, J. Geophys. Res., 101, 25,321-25,336, doi:10.1029/96JB02095.

Day, S. M., L. A. Dalguer, N. Lapusta, and Y. Liu (2005), Comparison of finite difference and boundary integral solutions to three-dimensional spontaneous rupture, J. Geophys. Res., 110, B12307, doi:10.1029/ 2005JB003813.

Dieterich, J. H. (1979), Modelling of rock friction: 1. Experimental results and constitutive equations, J. Geophys. Res., 84, 2161-2168, doi:10.1029/JB084iB05p02161.

Dieterich, J. H. (1981), Constitutive properties of faults with simulated gouge, in Mechanical Behavior Crustal Rocks, Geophys. Monogr. Ser., vol. 24, edited by N. L. Carter et al., pp. 103-120, AGU, Washington, D. C.

Dieterich, J. H. (2007), Application of rate- and state-dependent friction to models of fault slip and earthquake occurrence, in Treatise on Geophysics, vol. 4, Earthquake Seismology, edited by H. Kanamori, pp. 107-129, Elsevier, Amsterdam.

Duan, B., and D. Oglesby (2005), The dynamics of thrust and normal faults over multiple earthquake cycles: Effects of dipping fault geometry, Bull. Seismol. Soc. Am., 95(5), 1623-1636, doi:10.1785/0120040234.

Dunham, M., P. Favreau, and J. M. Carlson (2003), A supershear transition mechanism for cracks, Science, 299, 1557-1559, doi:10.1126/ science. 1080650 .

Geubelle, P., and J. R. Rice (1995), A spectral method for 3D elastodynamic fracture problems, J. Mech. Phys. Solids, 43, 1791-1824, doi:10.1016/0022-5096(95)00043-I.
Harris, R. A., et al. (2004), The source physics of large earthquakes: Validating spontaneous rupture methods, Eos Trans. $A G U, 85(47)$, Fall Meet. Suppl., Abstract S12A - 05.

Harris, R. A., et al. (2008), The SCEC/USGS dynamic earthquake-rupture code verification exercise, Seismol. Res. Lett., 80(1), 119-126, doi:10.1785/gssrl.80.1.119.

Hillers, G., Y. Ben-Zion, and P. M. Mai (2006), Seismicity on a fault controlled by rate- and state-dependent friction with spatial variations of the critical slip distance, J. Geophys. Res., 111(B0), B01403, doi:10.1029/2005JB003859.

Hori, T., N. Kato, K. Hirahara, T. Bada, and Y. Kaneda (2004), A numerical simulation of earthquake cycles along the Nankai trough, southwest Japan: Lateral variation in frictional property due to slab geometry controls the nucleation position, Earth Planet. Sci. Lett., 228, 215-226, doi:10.1016/ j.eps1.2004.09.033.

Kato, N. (2004), Interaction of slip on asperities: Numerical simulation of seismic cycles on a two-dimensional planar fault with nonuniform frictional property, J. Geophys. Res., 109, B12306, doi:10.1029/ 2004JB003001.

Kato, N., and T. E. Tullis (2003), Numerical simulation of seismic cycles with a composite rate- and state-dependent friction law, Bull. Seismol. Soc. Am., 93(2), 841-853, doi:10.1785/0120020118.

Kawasaki, I. (2004), Silent earthquakes occurring in a stable-unstable transition zone and implications for earthquake prediction, Earth Planets Space, 56, 813-821.

Lapusta, N., and J. R. Rice (2003), Nucleation and early seismic propagation of small and large events in a crustal earthquake model, J. Geophys. Res., 108(B4), 2205, doi:10.1029/2001JB000793.

Lapusta, N., J. R. Rice, Y. Ben-Zion, and G. Zheng (2000), Elastodynamic analysis for slow tectonic loading with spontaneous rupture episodes on faults with rate- and state-dependent friction, J. Geophys. Res., 105, 23,765-23,789, doi:10.1029/2000JB900250.

Liu, Y., and N. Lapusta (2008), Transition of mode II cracks from subRayleigh to intersonic speeds in the presence of favorable heterogeneity, J. Mech. Phys. Solids, 56, 25-50, doi:10.1016/j.jmps.2007.06.005.

Liu, Y., and J. R. Rice (2005), Aseismic slip transients emerge spontaneously in three-dimensional rate and state modeling of subduction earthquake sequences, J. Geophys. Res., 110, B08307, doi:10.1029/ 2004JB003424.

Marone, C. (1998), Laboratory-derived friction laws and their application to seismic faulting, Annu. Rev. Earth Planet. Sci., 26, 643-696, doi:10.1146/annurev.earth.26.1.643.

Palmer, A. C., and J. R. Rice (1973), The growth of slip surfaces in the progressive failure of overconsolidated clay slopes, Proc. R. Soc. London, Ser. A, 332, 527-548, doi:10.1098/rspa.1973.0040.

Perrin, G., J. R. Rice, and G. Zheng (1995), Self-healing slip pulse on a frictional surface, J. Mech. Phys. Solids, 43, 1461-1495, doi:10.1016/ 0022-5096(95)00036-I.

Rice, J. R. (1993), Spatio-temporal complexity of slip on a fault, J. Geophys. Res., 98, 9885-9907, doi:10.1029/93JB00191.

Rice, J. R. (2006), Heating and weakening of faults during earthquake slip, J. Geophys. Res., 111, B05311, doi:10.1029/2005JB004006.

Rice, J. R., and Y. Ben-Zion (1996), Slip complexity in earthquake fault models, Proc. Natl. Acad. Sci. U.S.A., 93, 3811-3818, doi:10.1073/ pnas.93.9.3811.

Rice, J. R., and A. L. Ruina (1983), Stability of steady frictional slipping, J. Appl. Mech., 50, 343-349.

Rubin, A. M. (2008), Episodic slow slip events and rate-and-state friction, J. Geophys. Res., 113, B11414, doi:10.1029/2008JB005642.

Rubin, A. M., and J. P. Ampuero (2005), Earthquake nucleation on (aging) rate and state faults, J. Geophys. Res., 110(B1), B11312, doi:10.1029/ 2005JB003686.

Ruina, A. L. (1983), Slip instabilities and state variable friction laws, J. Geophys. Res., 88, 10,359-10,370, doi:10.1029/JB088iB12p10359. Segall, P., and A. Rubin (2007), Dilatancy stabilization of frictional sliding as a mechanism for slow slip events, Eos Trans. $A G U, 88(52)$, Fall Meet. Suppl., Abstract T13F-08.

Shibazaki, B., and M. Mastsu'ura (1992), Spontaneous processes for nucleation, dynamic propagation, and stop of earthquake rupture, Geophys. Res. Lett., 19, 1189-1192, doi:10.1029/92GL01072.

Shibazaki, B., and T. Shimamoto (2007), Modelling of short-interval silent slip events in deeper subduction interfaces considering the frictional properties at the unstable-stable transition regime, Geophys. J. Int., 171, 191-205, doi:10.1111/j.1365-246X.2007.03434.x.

Suppe, J., and L. Yue (2007), Pore-fluid pressures and crustal strength, Eos Trans. $A G U, 88(52)$, Fall Meet. Suppl., Abstract T41F-01.

Tse, S. T., and J. R. Rice (1986), Crustal earthquake instability in relation to the depth variation of frictional slip properties, J. Geophys. Res., 91, 9452-9472, doi:10.1029/JB091iB09p09452. 
Zhang, H. M., and X. F. Chen (2006a), Dynamic rupture on a planar fault in three-dimensional half-space - I. Theory, Geophys. J. Int., 164, 633-652, doi:10.1111/j.1365-246X.2006.02887.x.

Zhang, H. M., and X. F. Chen (2006b), Dynamic rupture on a planar fault in three-dimensional half-space - II. Validations and numerical experiments, Geophys. J. Int., 167, 917-932, doi:10.1111/j.1365-246X.2006.03102.x.
Ziv, A., and A. Cochard (2006), Quasi-dynamic modeling of seismicity on a fault with depth-variable rate- and state-dependent friction, J. Geophys. Res., 111, B08310, doi:10.1029/2005JB004189.

N. Lapusta and Y. Liu, Division of Engineering and Applied Science, California Institute of Technology, 1200 East California Boulevard, Pasadena, CA 91125, USA. (lapusta@caltech.edu; yil@caltech.edu) 